\title{
Near-infrared light-mediated rare-earth nanocrystals: recent advances in improving photon conversion and alleviating the thermal effect
}

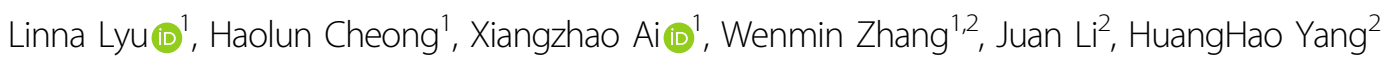 \\ Jun Lin ${ }^{3}$ and Bengang Xing ${ }^{1,2}$
}

\begin{abstract}
With the rapid development of nanotechnology, the unique rare-earth lanthanide-doped upconversion nanocrystals (UCNs), which can convert tissue-penetrable near-infrared (NIR) photonic irradiation into ultraviolet, visible, and NIR emissions, have a significant potential in bioimaging, diagnosis, and therapy, as well as in photovoltaic systems and optical data storage. Despite the promising achievements made in the past decade, critical challenges associated with low upconversion efficiencies and the overheating effect induced by NIR laser-irradiation still remain in the biomedical fields. In high demand are more well-defined material design and unique structural modifications that are capable of solving these technical concerns and promoting such promising NIR light-mediated upconversion nanocrystals for their further application in the medical sciences. Recent advances in upconversion nanomaterials have witnessed a tremendous development towards enhancing their photon conversion efficiency, which provides great opportunities in expanding the potential of the UCNs in bioimaging diagnosis and anticancer therapy. Hence, this review is mainly focused on summarizing the fundamental principles and strategies that improve upconversion luminescence and the approaches to reduce the local thermal effect on the basis of a rational design of UCNs. In addition, the future perspectives in the development of UCNs for biomedical applications are also proposed.
\end{abstract}

\section{Introduction}

The future of medicine lies in the diagnosis of diseases in their early stages and personalized treatments by integrating the individual variability in the medical profiles of patients ${ }^{1}$. Such a revolutionary paradigm requires precision medicine and a systematic understanding of detailed cellular functions, biological processes, and metabolic pathways associated with various diseases (such as cancers, diabetes, heart disorders, and neurodegenerative diseases)

Correspondence: Bengang Xing (Bengang@ntu.edu.sg)

'Division of Chemistry and Biological Chemistry, School of Physical \&

Mathematical Sciences, Nanyang Technological University, Singapore 637371,

Singapore

${ }^{2}$ College of Chemistry, Fuzhou University, Fuzhou, Fujian 350116, China

Full list of author information is available at the end of the article. in human beings and other living systems ${ }^{2,3}$. Recent advances in science and technology have witnessed molecular imaging as a key representative in the support of such new concepts of precision medicine in clinical trial designs $^{4,5}$. Thus far, molecular imaging has been well recognized as a powerful technique in the comprehensive evaluation of the essential biomolecules including nucleic acids, proteins, carbohydrates, lipids and their metabolites, as well as non-invasively visualization of the cellular functions or the biological processes in multiple biological systems, from cells to tissues and complex organisms in vivo ${ }^{6-8}$. More importantly, molecular imaging can also improve every aspect of precise diagnosis and treatment in clinical practice? 
Among the various imaging modalities, fluorescenceimaging strategy has attracted considerable attention, mainly owing to its distinctive advantages of ease of use, high sensitivity, good capability of providing real-time visualization of specific molecular targets and simultaneously monitoring of therapeutic intervention in vitro and in vivo, or even in patients ${ }^{10,11}$. Generally, the essential prerequisites to guarantee successful fluorescence imaging in biomedical and pre-clinical applications will greatly rely on the rational design of unique contrast agents. Until now, numerous fluorescent agents including organic fluorophores, genetically encoded fluorescent proteins, and quantum dot (QD) nanocrystals have been extensively explored for effective biosensing and real-time imaging of living species in vitro and in vivo ${ }^{12-15}$. Unfortunately, most of these conventional imaging agents utilize Stokes-shifted emission under light irradiation in the short-wavelength region (e.g., the ultraviolet (UV) and visible regions), which could potentially induce photoreactions in biomolecules including proteins or nucleic acids, and are thus harmful to living cells ${ }^{16}$. Moreover, the UV or visible light could be easily absorbed and scattered by certain biological tissues (such as fur, skin, and tissues), thus, leading to high auto-fluorescence, low signal-tobackground ratio, and low tissue penetration ${ }^{17,18}$. In addition, the potential toxicity of heavy-metal-based QDs would be one serious concern for bioimaging. Furthermore, although the utilization of near-infrared (NIR) fluorescent agents has increased, most of these dyes suffer from limited signal-to-noise ratio, a lesser Stokes shift, short lifetime, and poor photostability ${ }^{19}$. Therefore, the development of unique and reliable contrast agents that exhibit a high photostability, lack of toxicity, and long lifetime as well as deep tissue penetration properties are highly desirable. This remains a large technical challenge.

Recently, NIR light-mediated lanthanide-doped upconversion nanocrystals (UCNs), with their fascinating photon capability to convert NIR light illumination (e.g., at $808 \mathrm{~nm}$ or $980 \mathrm{~nm}$ ) into multiple emissions ranging from the UV to NIR regions through intermediate long-lived electronic states of lanthanide ions, are emerging as a new generation of luminescent materials and have attracted extensive interest among biomedical researchers ${ }^{20-24}$. Compared with traditional down-conversion fluorescent organic fluorophores or QDs, NIR light-mediated UCNs exhibit attractive advantages that make them well-suited for use in biomedical areas. For bioimaging, the obvious advantages are large anti-Stokes shifts allowing us to easily separate the emission from the excitation wavelength, narrow emission bandwidths making it suitable for multiplexed imaging, low autofluorescence background producing an improved signal-to-noise ratio, and high resistance to photobleaching allowing ease of long-term repetitive imaging ${ }^{25-27}$. In addition, the lower background and light scattering that allows for deep tissue light penetration will also cause UCNs to be attractive contrast agents for in vivo imaging ${ }^{28}$. Most importantly, UCNs with different dopants (e.g., Gd and Sm) could combine upconversion luminescent imaging with other imaging modalities such as computed tomography $(\mathrm{CT})^{29}$, magnetic resonance imaging $(\mathrm{MRI})^{30}$, positron emission tomography $(\mathrm{PET})^{31}$, and single photon emission tomography $^{32}$ for multimodality imaging ${ }^{33}$. In this regard, the use of NIR-triggered UCNs can greatly satisfy these demanding requirements, and have found various biomedical applications such as in vivo tumor imaging, multimodal animal imaging, multiplexed detection of analytes, remote-controlled drug delivery, cancer therapy, UCNs-mediated optogenetics, and so on (Fig. 1) ) $^{34-38}$.

Despite remarkable achievements in the past decade, some technical challenges associated with UCNs remain as concerns that may significantly restrict their further development with respect to wide-spread bioapplication in vitro and in vivo. One notable concern that may potentially affect substantial usage of UCNs lies in their low upconversion emission efficiency, which is largely due to the low extinction coefficients of lanthanide dopants, nanosize-induced surface-related quenching effects, and undesired interactions between lanthanide ions ${ }^{25,39}$. On the other hand, currently, most of the commonly used UCNs usually respond to the NIR laser irradiation (e.g., at $980 \mathrm{~nm}$ ), which overlaps the maximum absorption peak of water molecules, one of the most abundant NIR absorber in living systems ${ }^{40}$. Continuous laser irradiation at $980 \mathrm{~nm}$ to trigger the upconversion process would inevitably cause non-specific absorption by water molecules, thus, leading to local overheating effects. Such an unavoidable thermal effect may induce cell changes including loss of cell membrane asymmetry, nuclear fragmentation, and chromosomal DNA fragmentation, therefore, resulting in cell and tissue damage ${ }^{41}$. This non-negligible risk creates another technical obstacle for the in vivo applications of these NIR light-emitting materials. Regarding these issues, how to boost the upconversion luminescence efficiency of UCNs and minimize NIR laser-induced tissue damage will be of great significance in promoting fundamental research and broadening the applicability of lanthanidedoped nanomaterials.

So far, there have been a great number of excellent reviews of UCNs that emphasize the synthesis (e.g., size, morphology, and structure control $)^{27,42}$, tuning of optical properties $^{43,44}$, surface modification ${ }^{45}$, and their wide applications in biosensing, multimodal imaging, drug delivery, and light-controlled therapy (Fig. 1) 18,23,26,46-48. However, the technical challenges such as the low quantum yield and NIR laser-induced tissue overheating effect still need to be addressed. Hence, in this article, we mainly summarize principles and strategies regarding the 


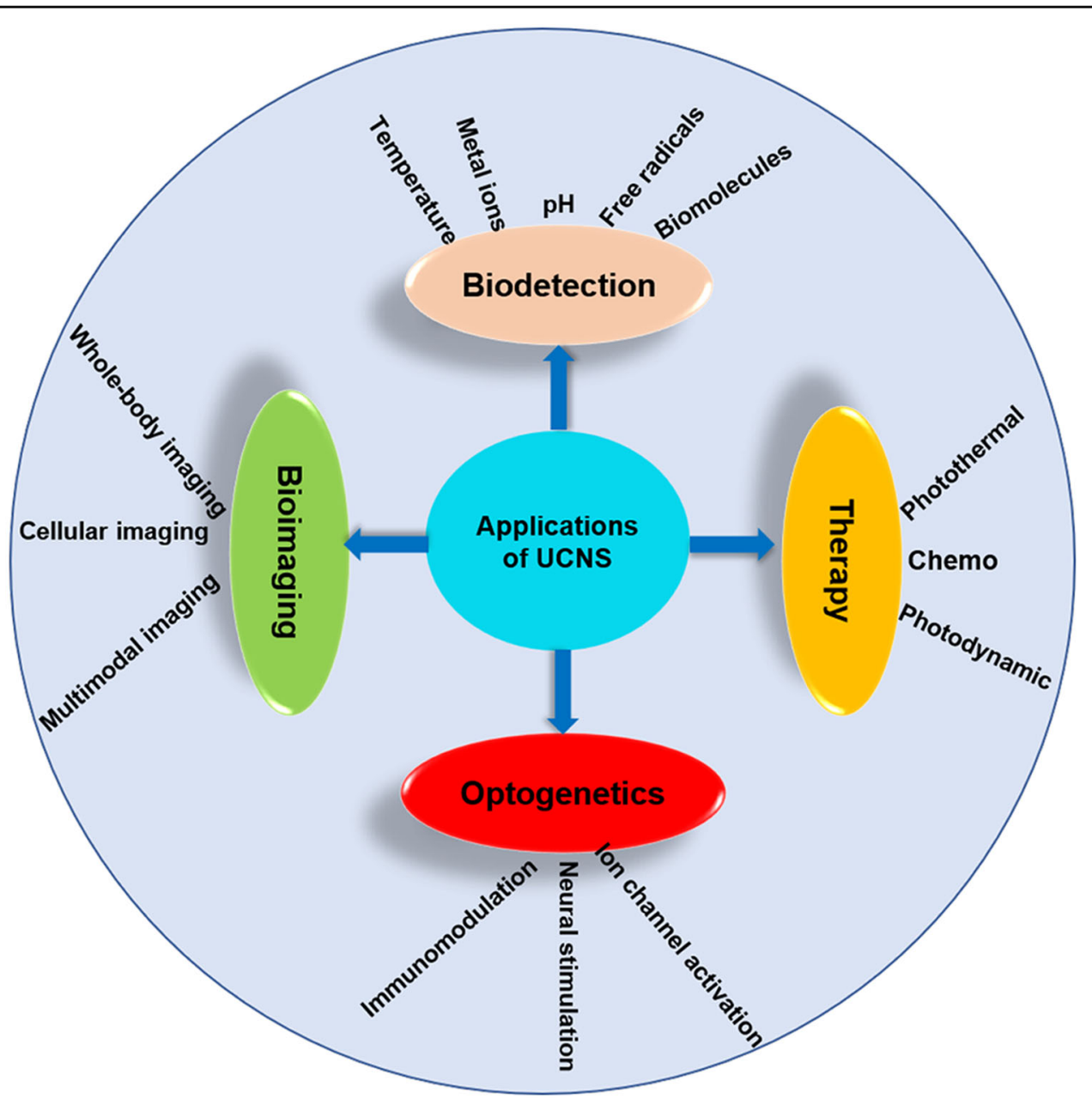

Fig. 1 The biomedical applications of upconversion nanocrystals: Biodetection, bioimaging, optogenetic and therapetic applications

improvement of upconversion luminescence as well as strategies to minimize the laser-induced overheating effect (Fig. 2). These improvement approaches provide an exciting opportunity to facilitate further development of NIR light-mediated UCNs as contrast agents in deep tissue imaging, high-sensitivity bioanalysis, remote activation of biomolecules, and so on. In addition, the future perspective of lanthanide-doped UCNs is also discussed.

\section{Strategies for enhancing photon upconverting efficiency}

With the development of lanthanide-doped nanocrystals, there recently have been many significant advances in synthesis techniques, size/morphology control and color tuning as well as functionalization modification of these upconverting nanoparticles for versatile biomedical applications. However, the low upconversion efficiencies remain the technical challenges in the design of welldefined UCNs. Therefore, to improve the practicality of upconversion nanomaterials in biomedical fields, it is essential to boost their upconversion luminescence. Hence, in this section, rational engineering of UCNs through crystal-lattice modification, energy-transfer manipulation, and design of core-shell structures as well as ingenious integration of lanthanide-based nanocrystals with other materials including plasmonic nanomaterials and organic dyes to increase the efficiency of photon upconversion will be discussed.

\section{Improving UC luminescence through alteration of the crystal field}

A notable reason for the low quantum efficiencies of UCNs is due to the low extinction coefficient of lanthanide dopants. Towards this end, one efficient strategy to improve the upconversion efficiency of UCNs would be the possibility of manipulation of the host matrix lattice. In general, the upconverted luminescence originates from the electronic transition within the $4 f$ orbital configuration of the lanthanide ions, where the parity-forbidden $f-f$ transitions become parity-allowed transitions through the 


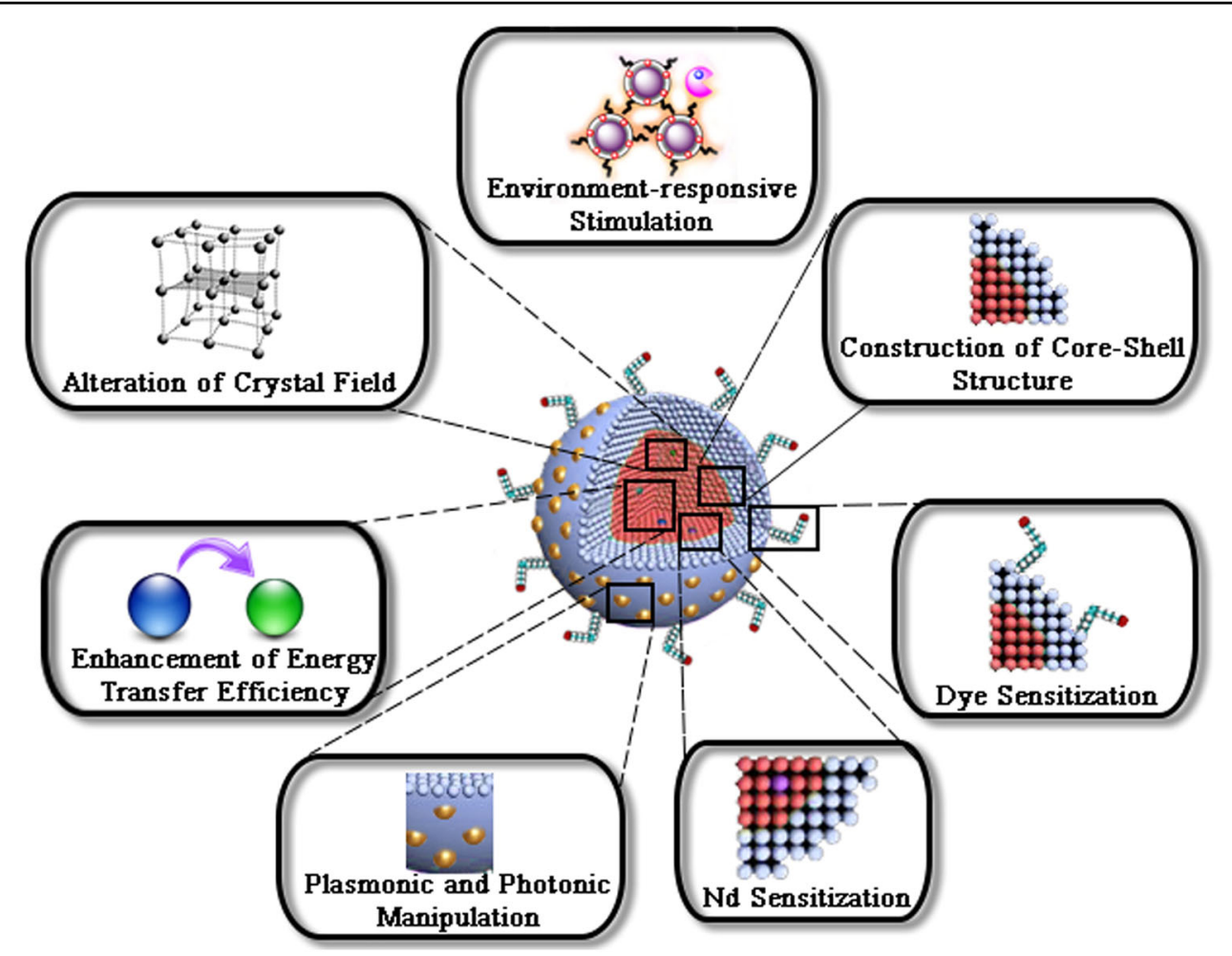

Fig. 2 Illustration of the main strategies for enhancing upconversion luminescence in lanthanide-doped UCNs

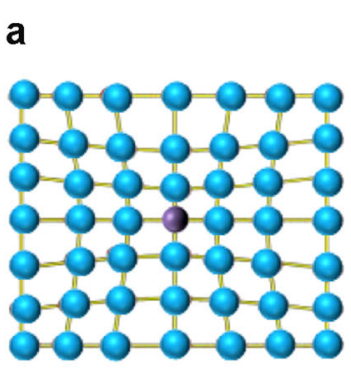

Host atom b

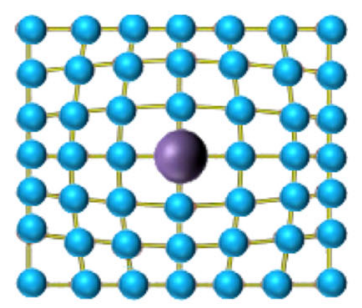

Dopant
C $980 \mathrm{~nm}$

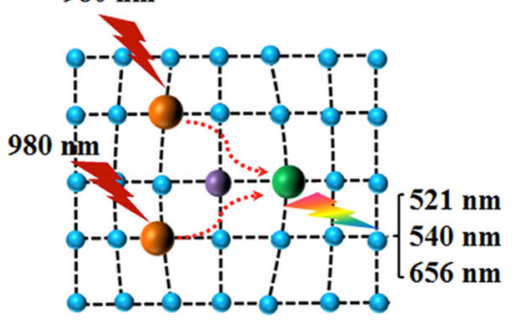

$\mathrm{Yb}^{3+}$ atom
$\mathrm{Er}^{3+}$ atom

Fig. 3 Crystal lattice contraction (left) and expansion (right) as a result of the substitution of a host atom with a dopant of varied size. Adapted with permission from Han et al. ${ }^{25}$ (copyright 2014 WILEY-VCH Verlag GmbH \& Co. KGaA, Weinheim)

intermixing of the $f$ states with high electron configuration. Hence, by influencing the structure of the crystal lattices, a probable improvement of the emission intensity of NIR light-mediated upconversion may occur, as the electric dipole transitions are allowed (Fig. 2).

The crystal field of photon-converted nanocrystals can be modified by doping with various ions, and the differences in the size of the doped ions can influence the coordination environment in the matrix (Fig. 3$)^{25}$, which results in an asymmetrical crystal field formation. The asymmetrical structural change in the crystal lattices will promote the intermixing of the $4 f$ energy levels of the lanthanide ions with high electronic configuration, thus, enabling the enhancement of the upconversion luminescence. Notably, a higher degree of asymmetry within the crystal field will consequently increase the upconverting efficiency. For example, early studies had validated the feasibility of a lithium-doping approach for improved upconversion emission ${ }^{49-52}$. Inspired by these pioneering studies, Zhao's group recently demonstrated the usage of $\mathrm{Li}^{+}$as a doping element in $\mathrm{GdF}_{3}: \mathrm{Yb} / \mathrm{Er}$ lanthanide nanocrystals, which were capable of enhancing the red emission upon $980 \mathrm{~nm}$ laser illumination, and thus indicated better tissue-penetration depth during fluorescence 

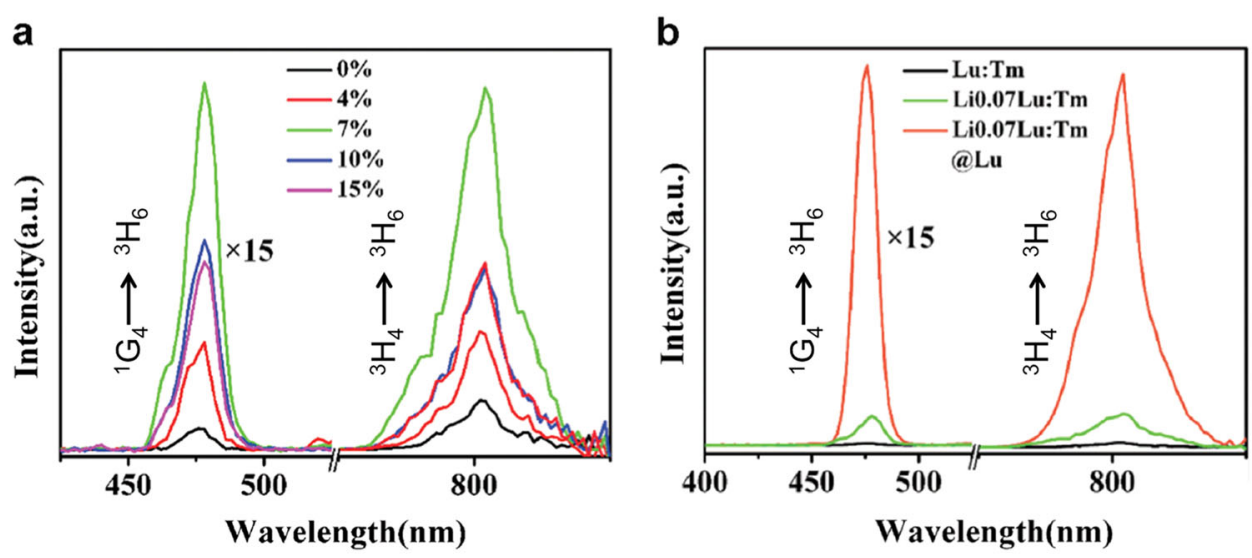

C
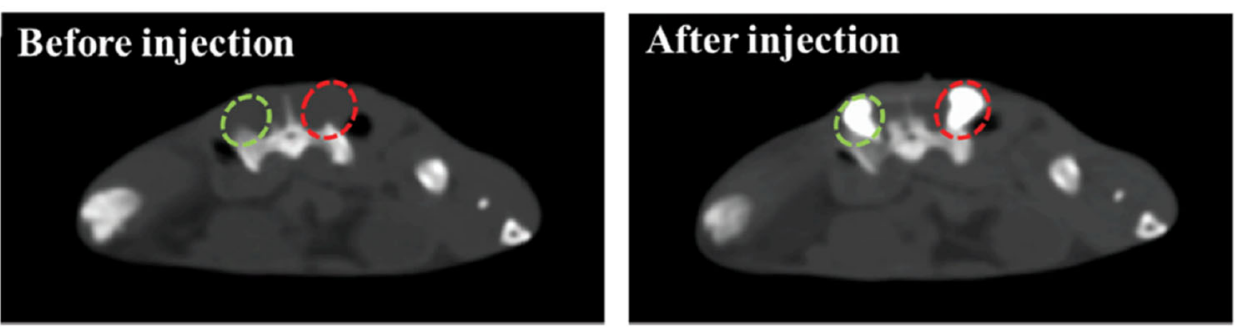

Fig. 4 Upconversion emission spectra of $\mathrm{Li}^{+}$-doped $\mathrm{NaLuF}_{4}: \mathrm{Yb}^{3+}, \mathbf{T m}^{3+}$ UCNs: a Different concentration of $\mathrm{Li}^{+}$dopant, $\mathbf{b}$ core-shell formation, c CT images of SD mouse injected with both COC-Li0.07Lu:Tm@Lu NPs (green circle) and iopromide (red circle). Adapted with permission from Hu et al. ${ }^{54}$ (copyright 2017 Royal Society of Chemistry)

imaging compared to the conventional upconversion luminescence at short-wavelength green and blue color windows. Moreover, the $\mathrm{GdF}_{3}$ host material also exhibited a high T1 MRI contrast effect, thus, allowing the UCNs to work as a dual-modality probe for optical and MRI imaging of cancer cells in vitro and in vivo ${ }^{53}$. In the same period, $\mathrm{Hu}$ et al. also developed a type of $\mathrm{Li}^{+}$-doped $\mathrm{NaLuF}_{4}: \mathrm{Yb}, \mathrm{Tm}$ UCNPs to amplify the upconversion emission for deep-tissue fluorescence imaging (Fig. 4$)^{54}$. Through the effect of $\mathrm{Li}^{+}$doping, an 18-fold increase in UC emission was observed. Additionally, the heavy atom effect of $\mathrm{Lu}^{3+}$ ions within the UCNPs nanostructures permits X-ray CT imaging, therefore providing a great potential in bio-multimodal tumor diagnoses in vitro and in vivo.

Apart from $\mathrm{Li}^{+}$ions, some other transitional metals have also been proposed as effective dopant elements in enhancing the UC luminescence. Usually, the $d$ orbitals in transitional metals indicate a larger radial size than the $f$ orbitals of the lanthanides. When they were doped into the host lattices of rare-earth lanthanides, these transitional metals will strengthen the electron-phonon coupling, and thus significantly enhance susceptibility to crystal-field perturbation. So far, several transitional metals such as $\mathrm{Sc}^{3+}, \mathrm{Zn}^{2+}, \mathrm{Bi}^{3+}, \mathrm{Fe}^{3+}$ have been reported to induce significant improvement in upconversion efficiency when doped into a host lattice comprising lanthanide activators ${ }^{55-57}$. For instance, by following this strategy, Ramasamy et al. demonstrated the possibility of greatly increasing the emission intensities of the green and red emissions by 34-fold and 30-fold, respectively, upon the incorporation of $\mathrm{Fe}^{3+}$ ions into $\mathrm{NaGdF}_{4}: \mathrm{Yb} / \mathrm{Er}$ particles ${ }^{58}$. Additionally, after covalent linking of folic acid on the surface of the UCNs as a targeting ligand, such unique nanomaterials could serve as contrast agents to perform in vivo tumor imaging (Fig. 4).

\section{Enhancing emission via manipulation of energy transfer}

Typically, other than altering the crystal field of UCNs to improve the UC luminescence through the host lattice manipulation approach, energy transfer modification has been well recognized as another commonly used strategy to enhance the upconversion efficiency. In general, the upconversion mechanism of NIR light-controlled UCNs is mainly based on the process of energy transfer upconversion, which usually involves radiative energy transfer between two different types of ions, namely, a sensitizer and an activator. Upon excitation by an NIR laser, a light-harvesting agent (acting as the sensitizer) will be pumped from the ground state to its excited state. 
Subsequently, the excitation energy is transferred from the sensitizer ion to the ground state of a neighboring activator, where activators will be excited to their emitting states and finally fulfill the upconverting emission ${ }^{22}$. However, due to the similarity in energy level between the ground and excited state, in the process of NIR laser conversion, the non-radiative energy transfer among the ions would be hard to avoid, which may thus affect the intensity of the converted luminescence. Such a nonradiative transfer mechanism usually involves fundamental multipolar and/or exchange interactions between two ions and is greatly dependent on their separation distance /, thus affecting the upconversion efficiency ${ }^{59}$. Hence, manipulating the concentrations of the lanthanide ions in the nanocrystals will influence the average distances within the ions, which therefore impact the non-radiative energy transfer.

In general, controlling the dopant concentration of activator ions will help to improve the light-mediated upconversion luminescence. A high doping level of activator, including ions such as $\mathrm{Tm}^{3+}, \mathrm{Er}^{3+}$ and $\mathrm{Ho}^{3+}$, has been proposed in the rational design of UCNs nanocrystals to maximize the energy transfer absorbed by the sensitizers. Nevertheless, such a high dopant concentration may also lead to an increase in cross-relaxation, which results in the self-quenching of the UC luminescence. To solve this issue, Zhao et al. ${ }^{60}$ recently reported the utilization of high-powered excitation irradiation combined with high activator concentration $\left(\mathrm{Tm}^{3+}\right)$ to regulate the UC emission after NIR laser irradiation of $\mathrm{NaYF}_{4}$ nanoparticles. These studies of UC luminescence clearly demonstrated that the use of high-power irradiance (e.g., $\sim 2.5 \times 10^{6} \mathrm{~W} \mathrm{~cm}^{-1}$ ) could alleviate the quenching effect of the high activator concentration (e.g., $8 \mathrm{~mol} \% \mathrm{Tm}^{3+}$ ) that is caused by cross-relaxation to achieve a 70-fold increase in luminescence. Furthermore, to achieve better upconversion efficiency through a high concentration of sensitizer ions $\left(\mathrm{Yb}^{3+}\right)$, Wang et al. ${ }^{61}$ proposed a new class of orthorhombic $\mathrm{KYb}_{2} \mathrm{~F}_{7}$ :Er nanocrystals, where the sensitizer ions are distributed into tetrad clusters. This unique lattice arrangement can effectively minimize the migration-mediated energy loss even with a high dopant concentration of $\mathrm{Yb}^{3+}$, thus, leading to an improvement in emission.

In addition to using conventional lanthanide ions to improve the energy transfer and increase the UC luminescence, it has been well known that the $d$ orbital of transition metals is able to tune the excited state of the lanthanide ions, which consequently demonstrates the potential in overcoming the phonon quenching effect, resulting in an increase in energy-transfer efficiency. Hence, the doping of transition metals in the nanoparticles will provide an alternative approach in intensifying the upconverted emission. For example, Dong et al. had demonstrated the usage of molybdenum $\left(\mathrm{Mo}^{3+}\right)$ as a dopant to enhance the blue and green emissions by several-fold ${ }^{62,63}$. They ascribed such enhancement in luminescence to the efficient energy transfer from the dimerized sensitizer, caused by the incorporation of $\mathrm{Mo}^{3+}$, to the activator ions. Similarly, UCNPs nanoparticle doped with the element manganese $\left(\mathrm{Mn}^{2+}\right)$ has also been reported to show the effective enhancement for the light-converted emission ${ }^{64-66}$.

Moreover, the exchange in excitation energy between the host lattices and the dopants can also help to increase the emission of the dopant ions at specific wavelength. Earlier studies had demonstrated that strong interaction between the host lattices $\left(\mathrm{Mn}^{2+}\right)$ and activator ions $\left(\mathrm{Er}^{3+}\right)$ in $\mathrm{MnF}_{2}: \mathrm{Yb} / \mathrm{Er}$ nanocrystal would result in an enhanced red-to-green emission ratio. Such unique phenomenon was mainly attributed to the relaxation of the excitation energy at ${ }^{4} \mathrm{~S}_{3 / 2}$ level of $\mathrm{Er}^{3+}$ to the lower ${ }^{4} \mathrm{~F}_{9 / 2}$ level, which was caused by the energy transfer of $\mathrm{Mn}^{2+}$ to $\mathrm{Er}^{3+67,68}$. Similar studies based on $\mathrm{KMnF}_{3}, \mathrm{NaMnF}_{3}$ and $\mathrm{NaYF}_{4}$ as host materials also displayed an intensified single-band emission with the incorporation of $\mathrm{Mn}^{2+}$ ions ${ }^{69-71}$.

Another notable energy transfer manipulation to improve the UC emission is the process based on the trapping of excitation energy within the nanoparticle structures. By introducing a trapping center with the addition of dopant, such a dopant process will confine the excitation energy and minimize migration-mediated energy loss, leading to the enhancement in energytransfer efficiency. For example, gadolinium $\left(\mathrm{Gd}^{3+}\right)$ is a typical dopant element commonly used in this approach mostly owing to its promising capacity to permit the trapping of the migrating energy by an activator ${ }^{72}$. As proof of concept, Zhou et al. demonstrated a $\mathrm{NaYF}_{4}$-based nanoparticle co-doped with $\mathrm{Gd}^{3+} / \mathrm{Yb}^{3+} / \mathrm{Er}^{3+}$ to enhance the UC emission. Furthermore, by taking advantage of the magnetic resonance properties of $\mathrm{Gd}^{3+}$, they established a promising multimodality probe that can be used for multimodality PET/MRI/UC luminescence imaging in vitro and in living animals (Fig. 5$)^{73}$. Using a similar principle of energy trapping, Liu's group recently reported the doping of $\mathrm{Tm}^{3+}$ in an $\mathrm{Er}^{3+}$-based nanocrystal could significantly enhance the upconversion emission (Fig. 6) ${ }^{74}$. The incorporation of $\mathrm{Tm}^{3+}$ ions will result in energy trapping, which will prevent distant energy migration and leads to a reduction in energy loss, thus, improving the upconversion efficiency of the nanocrystals.

\section{Amplifying upconverting efficiency by construction of core-shell structures}

Together with the strategies based on crystal-lattice modification and energy-transfer modification to increase upconversion emission, an alternative approach to enhance the luminescence efficiency in lanthanide-doped 

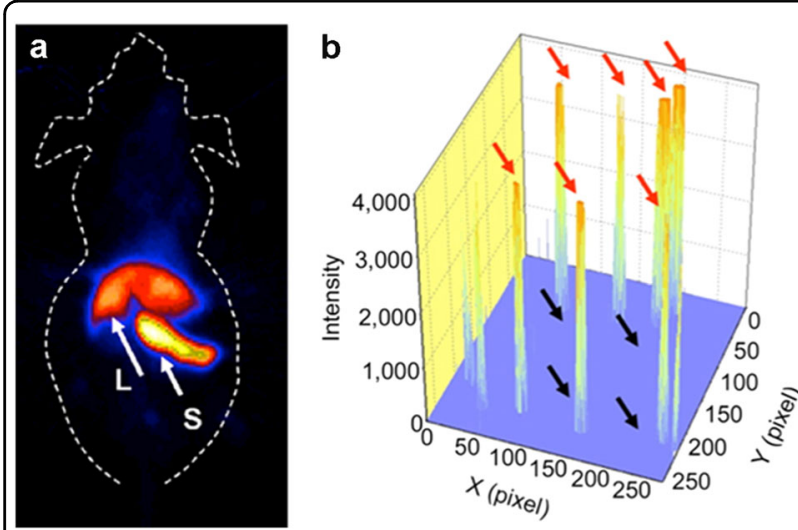

Fig. $5 \mathrm{Gd}_{3+} / \mathrm{Yb}_{3_{+}} / \mathrm{Er}_{3_{+}}$co-doped upconversion nanoparticles for multimodality imaging: a Whole-body two-dimensional projection, upconversion luminescence $(\mathrm{UCL})$ and bright-field images of the liver. b Quantization analysis of UCL signals of the liver section. Black arrows point to the background (counts $\sim 0$ ), and red arrows point to the signal (counts $>4095$ ). Adapted with permission from Zhou et al. ${ }^{73}$ (copyright 2011 Elsevier)

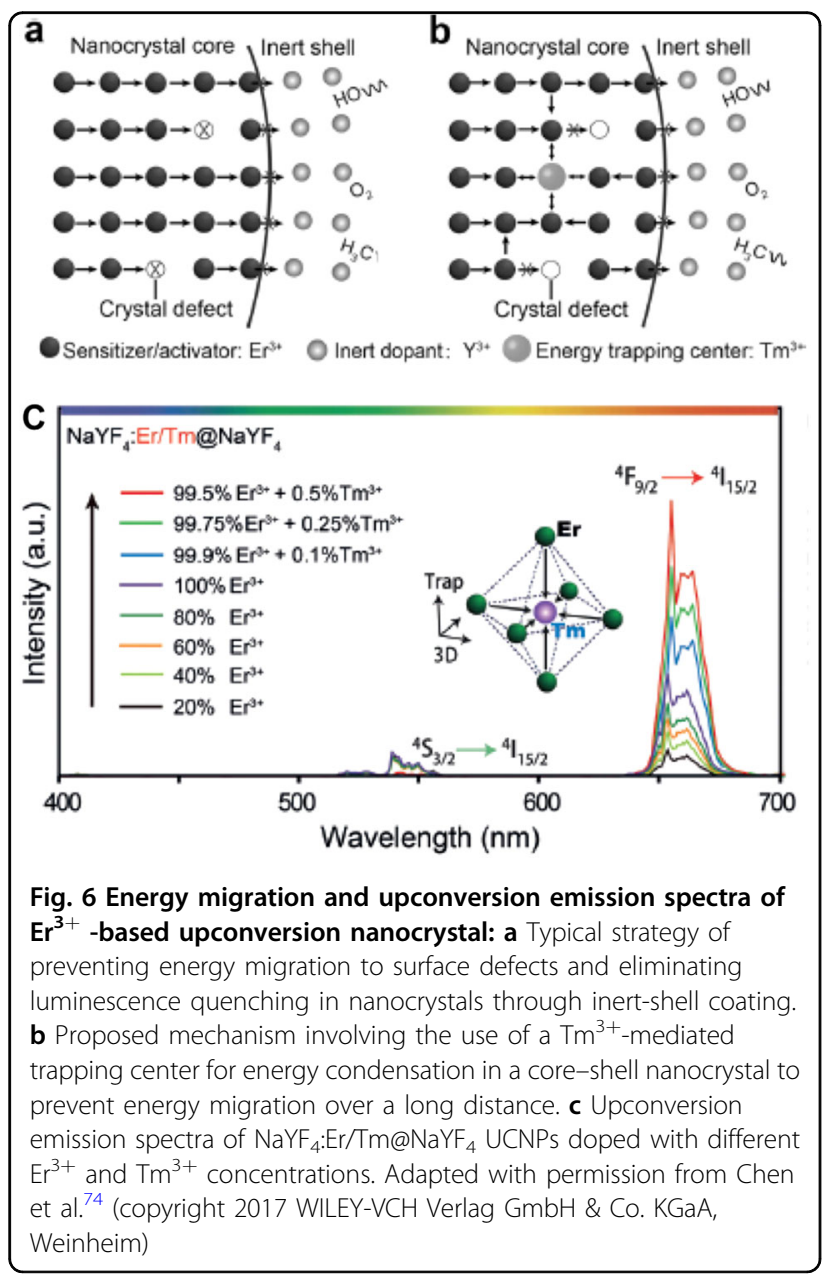

nanoparticles relies on the rational design of core-shell structures (Fig. 7). The surfaces of UCNs are highly related to the efficiency of UC luminescence, where the lanthanide dopants are exposed to surface quenching, which is usually induced by surface impurities, ligand and solvents through multi-phonon relaxation processes. Furthermore, these processes are prominent in the nanosized structures due to their high surface-to-volume ratio, which may lead to a strong surface quenching effect. Additionally, the excitation energy in the photoexcited dopants can undergo random migration and transfer to the surface-quenching sites, resulting in non-radiative relaxation. The suppression of surface-related quenching thus supplies a great possibility to effectively enhance the NIR light converting efficiency in UCN nanocrystals.

One typical strategy in suppressing surface quenching is the utilization of an optically inactive shell coating, which has identical materials to those of the core structure. The composition of shell/core structure not only provides a strong crystal field but also prevents the transfer of energy to the nanoparticle surface. The first demonstration of using an inactive shell composition to suppress passivation was reported by $\mathrm{Yi}$ and Chow, in which they investigated the effect of a $\mathrm{NaYF}_{4}$ shell coating on $\mathrm{NaYF}_{4}$ : Yb-based nanocrystals. Fluorescence imaging studies clearly showed a significant enhancement of approximately 30 -fold in the upconversion luminescence with the addition of the shell structure ${ }^{75}$. This method was further extended to other types of host materials, such as $\mathrm{NaGdF}_{4}$ and $\mathrm{KYF}_{4}$, and great success in the enhancement of the luminescence was achieved ${ }^{76-78}$.

In addition, an inert-shell coating composed of a difference chemical composition can also be constructed to significantly enhance the upconversion efficiency for the NIR light responsive nanomaterials. This type of core/ shell composition will improve the thermal stability of the nanoparticles and enable two or more imaging modalities in a single nanoparticle. As an example, Veggel's group proposed a rational design that coated the luminescence core $\mathrm{NaYF}_{4}: \mathrm{Yb} / \mathrm{Tm}$ with $\mathrm{NaGdF}_{4}$ as the shell ${ }^{79}$. With this design, enhanced UC luminescence could be easily achieved. Together with the paramagnetic property contributed by $\mathrm{Gd}^{3+}$ ions at the coating shell structure, such a unique platform could act as a dual-modality imaging probe for optical imaging and MRI for both in vitro and in vivo application.

Another strategy for enhancing the upconversion efficiency is to use an active shell coating over the UCNs (Fig. 7). The difference in this strategy compared to the inert-shell coating is that such a shell coating can be incorporated with a reasonable concentration of lanthanide dopants, which not only suppresses the surface quenching effect but also facilitates the energy transfer, thus making it possible to enhance the UC 


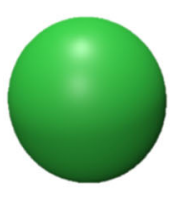

Core

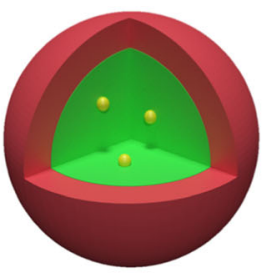

Inert shell

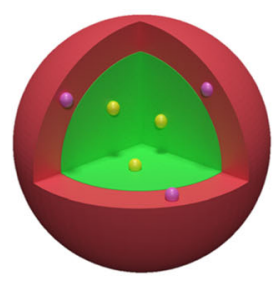

Active shell

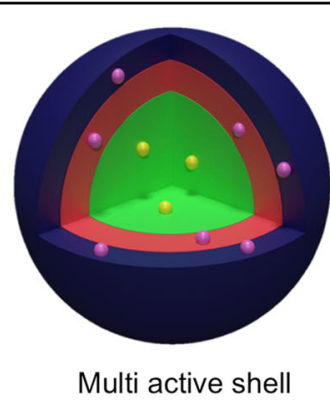

Sensitizer ions

Fig. 7 Modification of core-shell structures of nanoparticles to amplify upconverting efficiency: Schematic illustration of various core-shell structures

luminescence ${ }^{80}$. For example, Capobianco and co-workers reported the use of a $\mathrm{NaGdF}_{4}: \mathrm{Yb}$ active shell to coat the $\mathrm{NaGdF}_{4}: \mathrm{Yb} / \mathrm{Er}$ core nanoparticles, which exhibited a more effective UC emission enhancement compared to an inert shell ${ }^{81}$. Such luminescence enhancement could be mainly attributed to the insertion of the sensitizer $\mathrm{Yb}^{3+}$ ions into the shell coating, which preserved the excitation energy, and thus allowed the efficient energy transfer to the activators in the core structure. In another follow-up study, Zhang and Qian explored activators-encapsulated shells to improve the upconversion emission intensity ${ }^{82}$. Typically, they demonstrated that $\mathrm{NaYF}_{4} @ \mathrm{NaYF}_{4}$ nanoparticle doped with $\mathrm{Yb} / \mathrm{Tm}$ and $\mathrm{Yb} / \mathrm{Er}$ in the core and shell layer, respectively, displayed a significant enhancement in the emission intensity. Based on the same strategy, various core-shell materials could be constructed, and these have shown good success towards the process to enhance the emission intensity ${ }^{82-88}$.

Notably, Liu and Wang designed a novel core-shell nanostructure $\mathrm{NaGdF}_{4}: \mathrm{Yb}^{3+}, \mathrm{Tm}^{3+} @ \mathrm{NaGdF}_{4}: \mathrm{Ln}^{3+}(\mathrm{Ln}=$ $\mathrm{Tb}, \mathrm{Eu}, \mathrm{Dy}$, and $\mathrm{Sm}$ ), which displayed a unique UC process, namely, energy migration-mediated UC (EMU) (Fig. 8) ${ }^{89}$. In this NIR light-mediated UC process, the lanthanide ions designed for realizing EMU could be classified into four types: sensitizers, accumulators, migrators, and activators. Generally, the sensitizer ions collect the excitation energy and then subsequently transfer the energy to the accumulator ions to the excited state. Thereafter, the migrator ions will extract the excitation energy from the excited state of the accumulator ions, followed by random energy hopping within the migrator lattice. In addition, finally, the accumulator ions will trap the migration energy. The multilayered shell will spatially confine the sensitizer and accumulator as well as the activator ions in different layers, which will eliminate cross-relaxation and allow an efficient EMU process. Through this process, the surface quenching effect could be significantly inhibited, thus, enhancing the upconversion emission. Furthermore, such multiple-shell coatings could also enable a tunable emission by doping different activators on the outer shell. Very recently, Wang et al. further

realized enhanced multiphoton upconversion through spatial confinement of energy migration within the inner shell of $\mathrm{NaYF}_{4} @ \mathrm{NaYbF}_{4}: \mathrm{Tm} @ \mathrm{NaYF}_{4}$ core-shell-shell nanostructure. The upconversion emission spectrum of the nanoparticles showed that both violet and overall emissions surpassed that of the $\mathrm{NaYF}_{4} @ \mathrm{NaYbF}_{4}$ : $\mathrm{Tm} @ \mathrm{NaYF}_{4}$ counterparts with lower $\mathrm{Yb}^{3+}$ contents. Notably, a 45 -fold luminescent increment at $290 \mathrm{~nm}$ was observed when the $\mathrm{Yb}^{3+}$ concentration was increased to 99 mol\% (Fig. 9 ${ }^{90}$. In addition, by decreasing the inner shell thickness, they observed a steady enhancement of upconversion emission, especially the part in the $280-356 \mathrm{~nm}$ range. The mechanistic investigation revealed that the confined migration of excitation energy could prevent energy loss to the crystal lattice and increase the local density of excitation energy, thereby generating intense ultraviolet emissions on NIR light excitation. Recently, Jin and co-workers discovered that the emission efficiency of $\mathrm{Tm}^{3+}$-doped core-shell nanoparticles can be enhanced via the use of a laser cavity. With suitable control of the lasing conditions, upconversion efficiency for the 300-620 nm emission band of the $\mathrm{Tm}^{3+}$-doped nanoparticles under $976 \mathrm{~nm}$ excitation can be amplified by an order of magnitude over the case without a laser cavity ${ }^{91}$.

\section{Magnifying photon upconversion through plasmonic and photonic manipulation}

Another effective approach to achieve enhancement of upconversion emission is to use external field enhancement. One well-known method is to couple metal nanostructures to the UCN structures. Typically, when the metal-based nanostructures are irradiated with a beam of incident light, the excited electrons rapidly accumulate on their surface ${ }^{18}$. The collective oscillation of electrons on the metal surface is known as surface plasma resonance, which can generate large local electric fields in the vicinity 

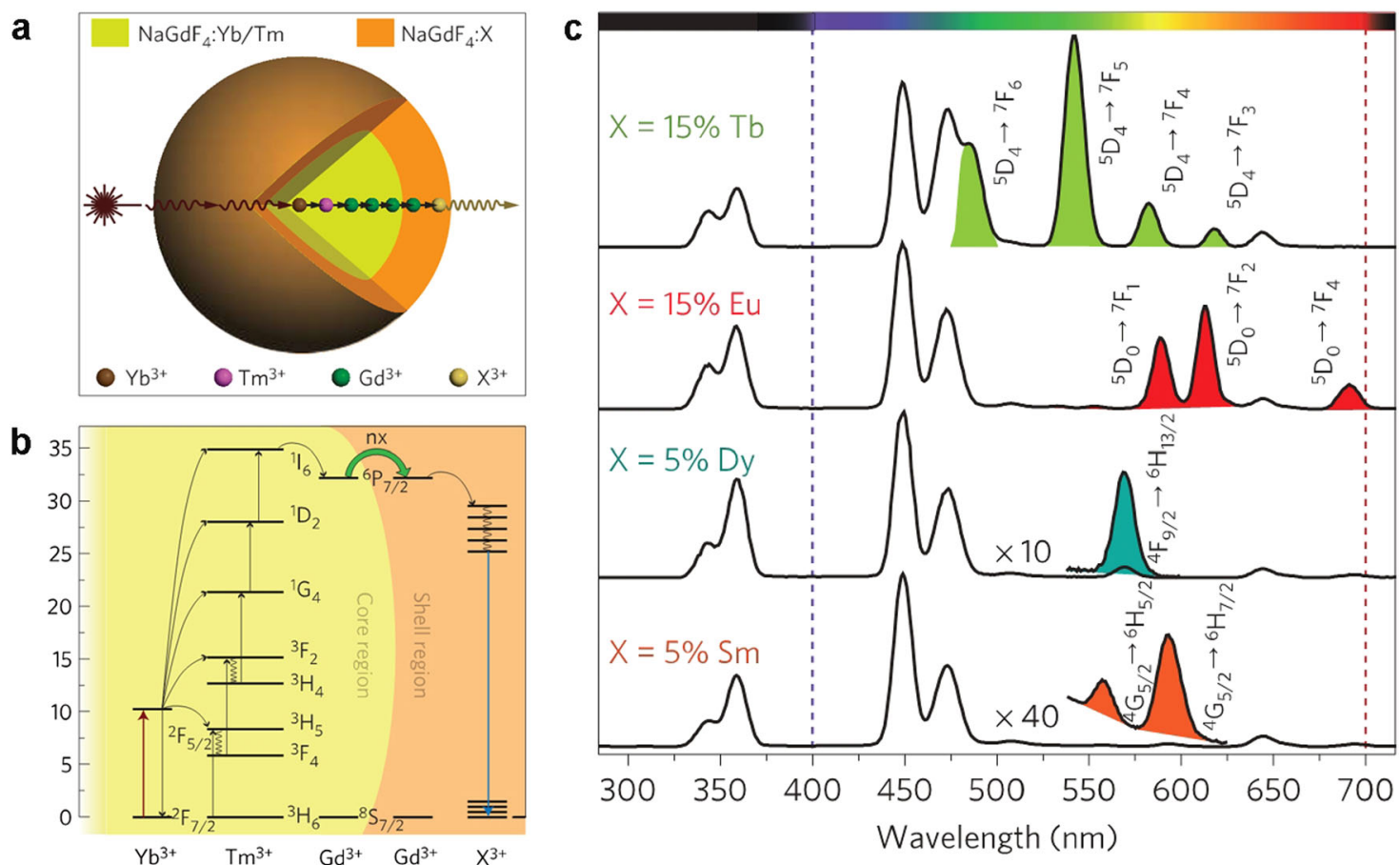

Fig. 8 Tuning upconversion through core-shell nanoparticles: a Schematic design of a $\mathrm{Ln}^{3+}$-doped $\mathrm{NaGdF}_{4} @ \mathrm{NaGdF}_{4}$ core-shell NP for EMU (X: activator ions). b Proposed energy transfer mechanism between lanthanide dopants. c Emission spectra of the as-prepared $\mathrm{NaGdF}_{4} @ \mathrm{NaGdF}_{4}$ core-shell nanoparticles doped with different activators (activator emissions are highlighted with color). Adapted with permission from Liu et al. ${ }^{89}$ (copyright 2011 Nature group)

of metal nanostructures ${ }^{92}$. Most importantly, such a local field enhancement effect can further increase the excitation and radiative decay rates of the lanthanide-doped $\mathrm{UCNs}^{93}$.

These metal-induced electric fields greatly depend on the composition, size, and morphology of the metal nanostructures $^{94}$. Therefore, it is highly important to optimize the plasmonic structure for enhancing upconversion luminescence. In this regard, various UCNs-metal geometric configurations have been constructed. Recently, Kagan et al. have fabricated a metaloxide-UCNP multilayered structure for amplification of luminescence in UCNs. Luminescence enhancements were found to be dependent on the thickness of the oxide spacer layer and the different types of metal NP (Fig. 10) $)^{95}$. Under the conditions of the largest enhancement in luminescence, the spacer thickness was found to be $5 \mathrm{~nm}$ for $\mathrm{Au}$ NPs and $10 \mathrm{~nm}$ for Ag NPs. Similarly, Zhang and coworkers utilized a 3D plasmonic architecture, the disk-coupled dots-on-pillar antenna array $\left(\mathrm{D}_{2} \mathrm{PA}\right)$, to regulate the NIR photon converted emissions in $\mathrm{NaYF}_{4}: \mathrm{Yb}^{3+} / \mathrm{Er}^{3+}$ doped nanocrystals. The plasma frequency could be tuned by adjusting the height of the $\mathrm{SiO}_{2}$ pillars. When the pillar height was set to $75 \mathrm{~nm}$, a 310-fold luminescent increment was observed $^{96}$. Additionally, other Au- or Ag-containing plasmonic nanostructures like nanoshells, nanorods, nanowires, and nanohole arrays were also fabricated to achieve plasmonic-promoted upconversion emission ${ }^{97}$.

Recently, the coupling of the UCNs with photonic crystals (PCs) also has been exploited to magnify the upconversion emission. PCs are materials with periodic dielectric structures that can affect the propagation of electromagnetic waves and generate photonic bandgaps. Moreover, the resonance of the excitation wavelengths with PC modes could result in an enhanced electric field on the PC surface, which further leads to increased absorption of the nanomaterials on the surface of the PCs. Based on the unique properties of PCs, Niu and coworkers recently coupled $\mathrm{NaYF}_{4}$ nanoparticles on the surface of three-dimensional PCs (3D PCs) to achieve increased upconversion emission (Fig. 10d). Studies have shown that greater than a 30-fold UCL enhancement was observed for $\mathrm{NaYF}_{4}: \mathrm{Yb} / \mathrm{Er}$ and $\mathrm{NaYF}_{4}: \mathrm{Yb} / \mathrm{Tm}^{98}$. Mechanism studies revealed that the enhanced absorption in combination with both the strong coherent scattering and consequent-extraction effect mainly contributed to the emission enhancement of UCNPs on the PCs surface. Furthermore, by integrating this $3 \mathrm{D} \mathrm{PC} / \mathrm{NaYF}_{4}: \mathrm{Yb} / \mathrm{Tm}$ UCNP hybrid material with a carbon nanotube 

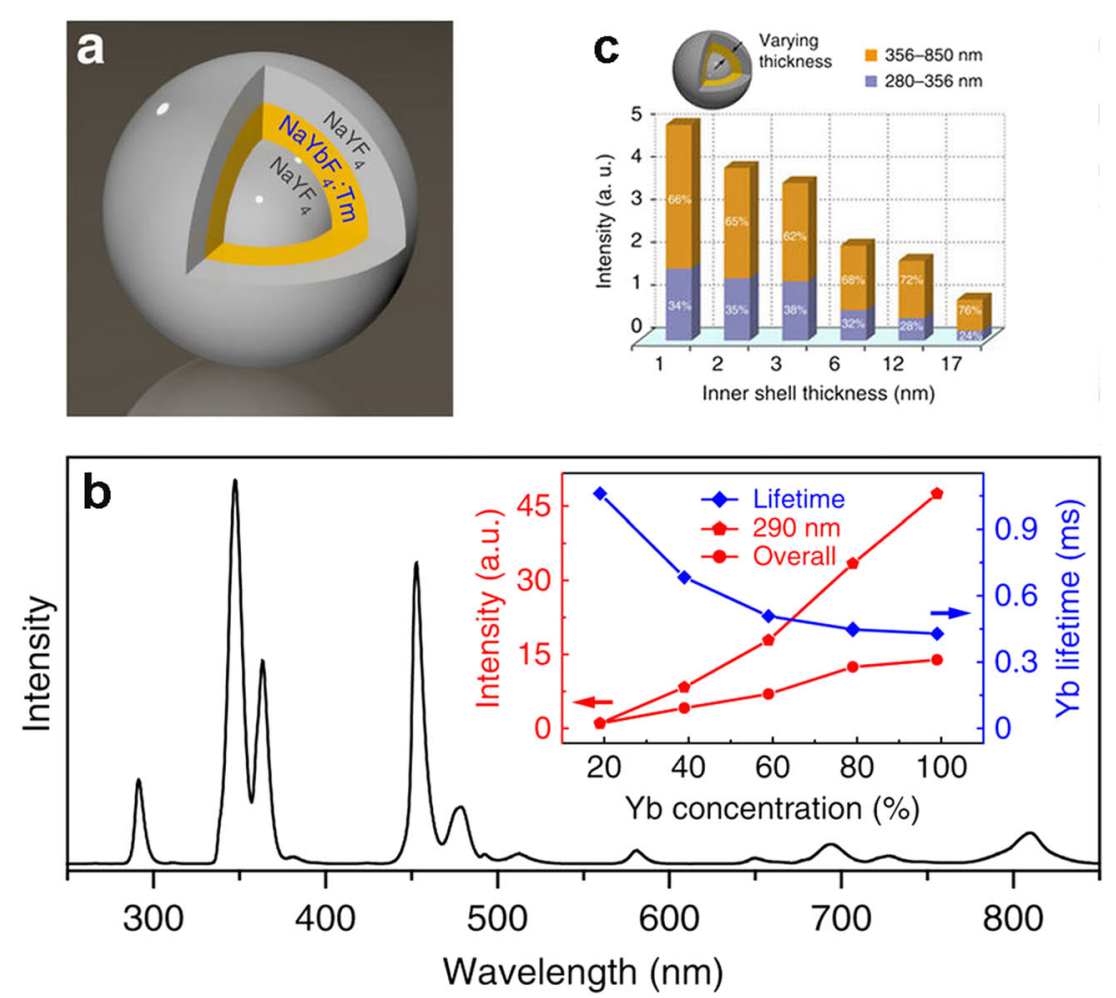

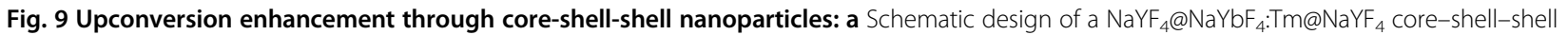
nanoparticle for confining the migration of excitation energy generated in the $\mathrm{Yb}^{3+}$ ions. $\mathbf{b}$ Upconversion emission spectrum of the nanoparticles under $980 \mathrm{~nm}$ excitation. Inset: ${ }^{2} \mathrm{~F}_{5 / 2}$ lifetime of $\mathrm{Yb}^{3+}$, emission intensity at $290 \mathrm{~nm}$ and integrated emission intensity over the $250-850 \mathrm{~nm}$ range versus dopant concentration of $\mathrm{Yb}^{3+}$, respectively. c Upconversion emission intensity versus inner shell thickness (1-17 nm). The emission intensities were calculated by integrating the spectral intensity of the emission spectra that are normalized to the absorption of $\mathrm{Yb}^{3+}$ at $980 \mathrm{~nm}$. Adapted with permission from Wang et al..$^{90}$ (copyright 2016 Nature group)

(CNT)-based phototransistor, the authors successfully achieved an amplification of the NIR light photoresponsivity tenfold greater than that of the CNT-based platform.

\section{Optimizing upconversion process via dye sensitization}

Although the various approaches through crystal lattice modification, energy transfer modulation, design of coreshell structures, and plasmonic enhancement have been well established to improve upconversion emission of UCNs, the inherently weak and narrow-band absorption of lanthanide ions remains the technical limitation, influencing their light-harvesting ability and thus, restricting their upconversion luminescence. Usually, such a major limitation is mainly caused by the parityforbidden nature of the $4 f-4 f$ electronic transitions of the doped lanthanide ions ${ }^{99}$. Generally, the sensitizers in UCNs could harvest NIR light irradiation and transfer energy to the activators $\mathrm{Ln}^{3+}$ to generate luminescence. However, the typically used $\mathrm{Yb}^{3+}$ sensitizer ions in UCN structures have a narrow absorption band (e.g., $980 \mathrm{~nm}$ ), with a small absorption cross-section $(\sim 1000-10,000$ times than those of organic dye molecules $)^{100}$, which significantly reduces the luminescent efficiency of UCNs . To address this fundamental limitation, organic dyes have been recently proposed as broadband sensitizers to improve the brightness of lanthanide-doped nanocrystals because of their large absorption cross-sections, increased light absorption ability and tunable excitation wavelength. In general, organic dyes acting as antennas could absorb NIR light in a broad and strong manner and transfer their excitation energy to the intermediate lanthanide sensitizers. The latter divert energy to the activator to produce a bright luminescence. By adopting this strategy, Zou and co-workers first reported the IR-806-sensisized $\mathrm{NaYF}_{4}$ : $\mathrm{Yb}^{3+} / \mathrm{Er}^{3+}$ nanoparticles that can absorb light over a broad wavelength range (from 650 to $850 \mathrm{~nm}$ ) and achieve a remarkable increase in luminescence intensity ${ }^{101}$. Later, a set of organic fluorophores was utilized to widen the photon absorption range and boost the upconversion efficiency of $\mathrm{UCNs}^{102,103}$.

In continuing research, the core/shell structures have been introduced to NIR dye-sensitized nanocrystals, aiming to solve the issues of surface-related luminescence quenching. In these dye-sensitized core/shell nanocrystals, the organic fluorophore molecules could absorb 


\section{a}

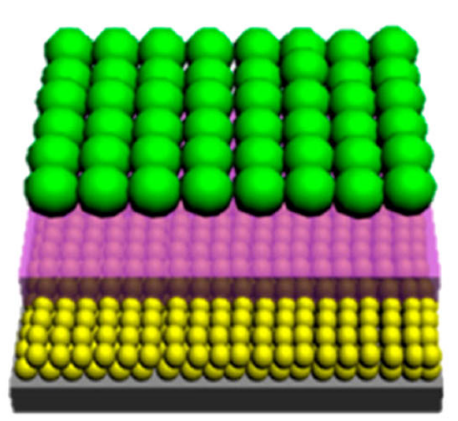

d

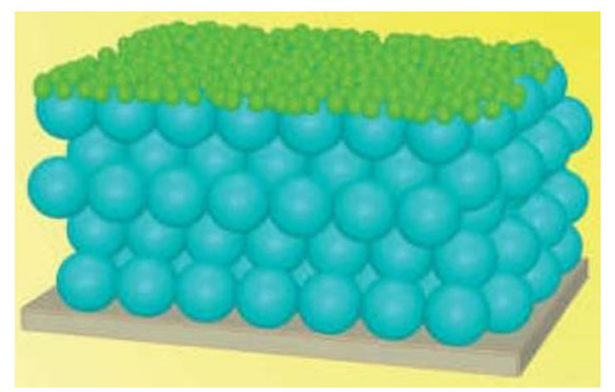

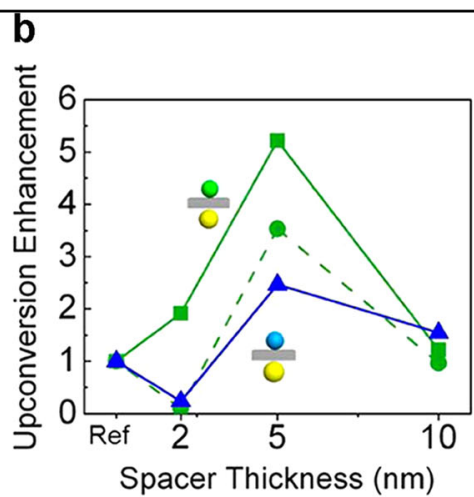

e

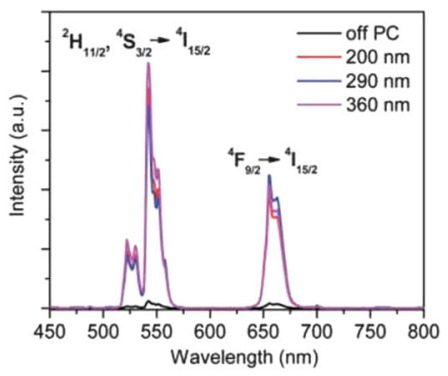

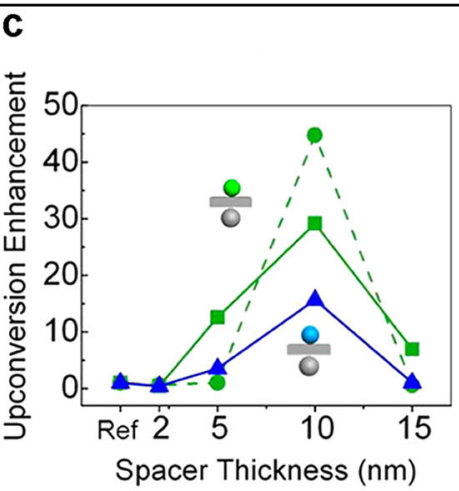

f

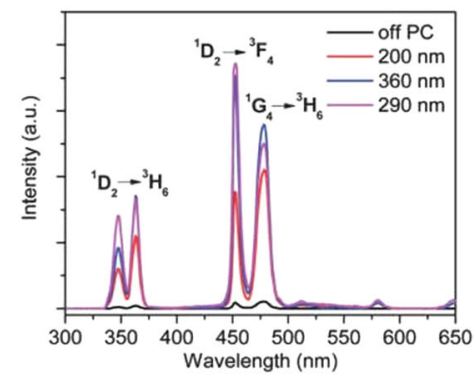

Fig. 10 Plasmonic and photonic manipulation of upconversion nanoparticles in enahancing upconverted emission: a Schematic structure of a spin-cast layer of $\mathrm{Au}$ or $\mathrm{Ag} \mathrm{NPs}$, a thin $\mathrm{Al}_{2} \mathrm{O}_{3}$ layer, and a monolayer of UCNs. Integrated area under the peak as a function of the $\mathrm{Al}_{2} \mathrm{O}_{3}$ layer thickness normalized to that of the pure UCN sample for (b) Au NPs and (c) Ag NPs. The green solid lines correspond to the $540 \mathrm{~nm}$ emission band

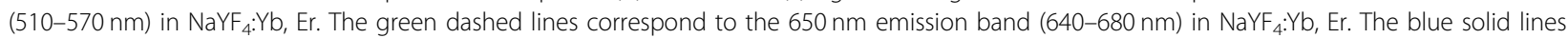
correspond to the $475 \mathrm{~nm}$ emission band $\left(461-490 \mathrm{~nm}\right.$ ) in NaYF $4: \mathrm{Yb}$, Tm. Adapted with permission from Kagan et al. ${ }^{95}$ (copyright 2012 American Chemical Society). d Schematic illustration of self-assembled photonic crystals with a layer of fluoride UCNs. Upconversion luminescence spectra of $\mathrm{NaYF}_{4}: \mathrm{Yb} / \mathrm{Er}(\mathbf{b})$ and NaYF$: \mathrm{Yb} / \mathrm{Tm}$ (e) UCNs on the surfaces of 3D PCs prepared with different polystyrene sphere sizes at 0.4 wt.\% concentration. Adapted with permission from Niu et a $\left.\right|^{98}$ (copyright 2014 Royal Society of Chemistry)

infrared light radiation and transfer the excitation energy to the intermediate sensitizers within the shell, which subsequently transfer energy to the nearby sensitizers in the core of the UCN structure. Finally, the energy could be trapped by a neighboring activator to produce upconversion (Fig. $11 \mathrm{a}, \mathrm{b})^{100}$. Such an energy-cascaded

conversion process offers a maximum overlap between the emission of an energy donor and the absorption of an energy acceptor owing to the small energy gap in each step, resulting in effective transfer of harvested light energy all the way down to the upconverting lanthanide ions in the core structure. Moreover, a core/shell design could avoid the exposure of the core to the surrounding luminescence deactivators (e.g., surface defects, remaining ligands, and solvents), leading to remarkable suppression of surface-related luminescent quenching in the core structure of the nanocrystals. As a result, the dyesensitized core/shell nanocrystals combine the advantages of both the core-shell structure and the "antenna" effect from the dye molecules, producing ultra-bright upconversion luminescence under excitation in a broad spectral range.
To date, several types of dye-sensitized core/shell nanocrystals have been successfully applied in biomedical fields. For example, Han et al. found that IR806-sensitized core/shell nanoparticles $\left(\beta-\mathrm{NaYF}_{4}: 20 \% \mathrm{Yb}^{3+}, 2 \% \mathrm{Er}^{3+} @ \beta\right.$ $\mathrm{NaYF}_{4}: 10 \% \mathrm{Yb}^{3+}$ ) exhibited an enhanced upconversion emission under $800 \mathrm{~nm}$ excitation (Fig. 11). By taking advantage of such a dye-sensitized core/shell system, they successfully achieved precise control of neuronal activation in the NIR range ${ }^{104}$. Recently, Li et al. also reported water-dispersible $\mathrm{Cy} 7$-sensitized $\mathrm{NaYF}_{4}: \mathrm{Yb}, \mathrm{Nd}$, $\mathrm{Er} @ \mathrm{NaYF}_{4}: \mathrm{Nd}$ nanocrystals with approximately a 17 -fold enhancement of upconversion luminescence, which was successfully applied in lymphatic imaging as the first example in the field ${ }^{105}$.

\section{Modulating UC emission by environment-responsive stimulation}

Unlike the typical strategies in modifying the UCNs mentioned earlier, some distinctive approaches through responsive stimulation have been recently proposed to promote the luminescence of UCNs. For instance, Xing et al. presented a unique microenvironment-sensitive 

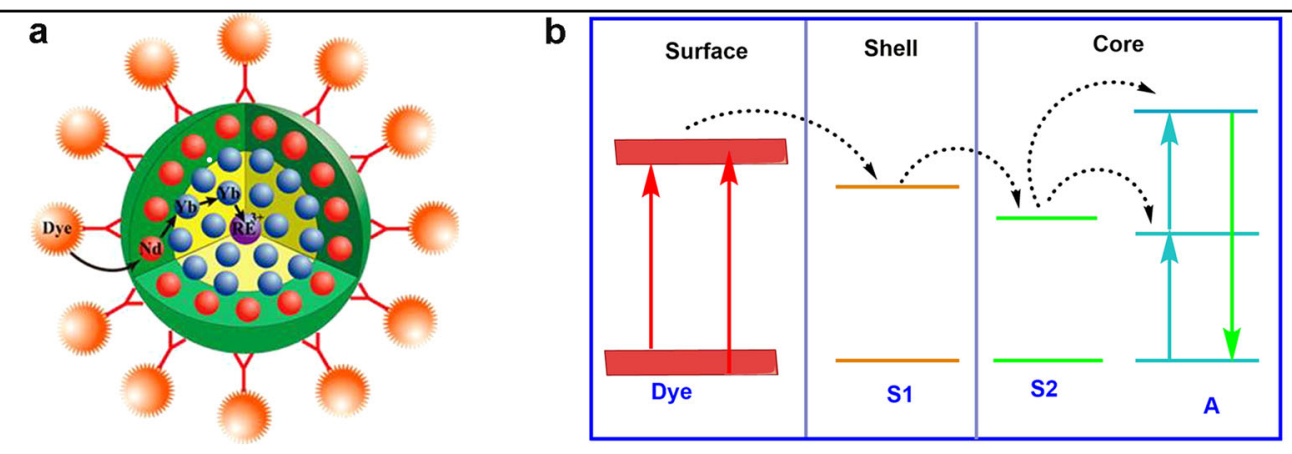

C

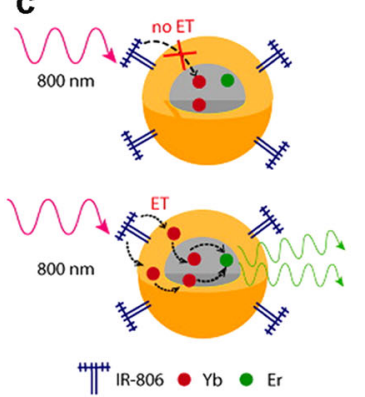

d

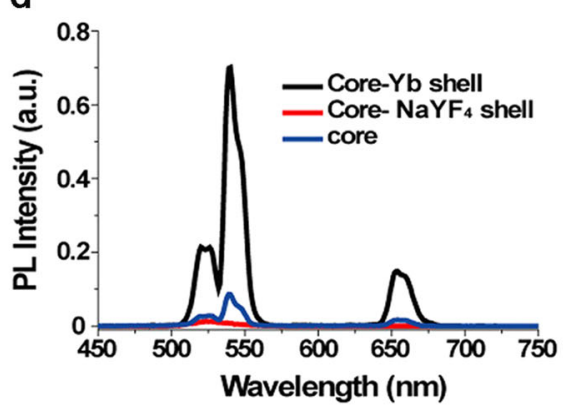

e

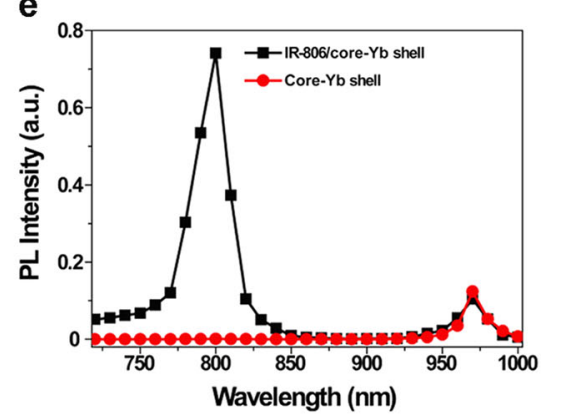

Fig. 11 NIR dye-sensitized modified upconversion nanoparticles: a, b Schematic illustrations of energy-cascaded upconversion in dye-sensitized core/shell nanocrystals (S1: type 1 sensitizer; S2: type 2 sensitizer; A: activator). Adapted with permission from Prasad et al. ${ }^{100}$ (copyright 2015 American Chemical Society). c Schematic showing the proposed energy transfer mechanism for core/shell UCNs without (top) and with (bottom) $\mathrm{Yb}^{3+}$ doping. $\mathbf{d}$ Emission spectra of IR-806-sensitized core only $\left(\beta-\mathrm{NaYF}_{4}: 20 \% \mathrm{Yb}^{3+}, 2 \% \mathrm{Er}^{3+}\right)$, core $/ \mathrm{NaYF}_{4}$ shell $\left(\beta-\mathrm{NaYF}_{4}: 20 \% \mathrm{Yb}^{3+}, 2 \% \mathrm{Er}^{3+} @ \beta-\mathrm{NaYF}_{4}\right)$, core $/ \mathrm{Yb}^{3+}$ shell $\left(\beta-\mathrm{NaYF}_{4}: 20 \% \mathrm{Yb}^{3+}, 2 \% \mathrm{Er}^{3+} @ \beta-\mathrm{NaYF}_{4}: 10 \% \mathrm{Yb}^{3+}\right)$. e Excitation spectrum of $\beta-\mathrm{NaYF}_{4}: 20 \% \mathrm{Yb}^{3+}, 2 \% \mathrm{Er}^{3+} @ \beta-\mathrm{NaYF}_{4}: 10 \% \mathrm{Yb}^{3+}\left(\mathrm{core} / \mathrm{Yb}^{3+}\right.$ active shell) nanoparticle with (black squares) and without (red circles) IR-806 dye sensitization. Adapted with permission from Han et al. ${ }^{104}$ (copyright 2016 American Chemical Society)

strategy for cross-linking of UCNs within tumor areas, leading to enhanced upconversion emission upon $808 \mathrm{~nm}$ laser excitation. In this pioneering work, the tumorspecific cathepsin protease could trigger the covalent cross-linking and accumulation of the peptidepremodified UCNs at the tumor region, which significantly magnify upconversion luminescence upon $808 \mathrm{~nm}$ laser irradiation (Fig. 12) ${ }^{106}$. Similarly, Liras and co-workers capped upconversion nanoparticles $\left(\beta-\mathrm{NaYF}_{4}\right.$ : $\left.\mathrm{Er}^{3+}, \mathrm{Yb}^{3+}, \mathrm{UCNs}\right)$ with $\mathrm{P}\left(\mathrm{MEO}_{2} \mathrm{MA}-\mathrm{co}-\mathrm{SEMA}\right)$ copolymers to form nanohybrids that exhibited an enhanced emission by up to a factor of ten, compared with that of their hydrophobic precursor in dichloromethane and even in water (a factor of two). Moreover, these polymercapped UCNs possessed thermoresponsive properties, and their emission was affected by temperature. Studies showed that these UCNs have a higher emission at $20^{\circ} \mathrm{C}$ than at $60{ }^{\circ} \mathrm{C}^{107}$.

\section{Strategies for minimizing the tissue-heating effect}

Though the low upconversion efficiency of UCNs can be improved through the aforementioned strategies to promote their extensive practical applications in living systems, one more challenge associated with the laserinduced tissue overheating effect heavily hinders their further exploration of pre-clinical applications. During the optical imaging in a biological system, the commonly used NIR laser irradiation (e.g., at $980 \mathrm{~nm}$ ) usually causes strong water absorption and thus, substantially induces a localized overheating effect, which may lead to obvious cell changes (e.g., cell shrinkage, protein denaturation, or chromosomal DNA fragmentation) and even terrible damage to normal tissues ${ }^{108}$. Therefore, it is also highly essential to optimize the excitation to an appropriate range for the purpose of minimizing this thermal issue, thus, making UCNs more suitable for biological applications.

One common strategy to overcome the issue of NIR laser-induced tissue damage is the introduction of $\mathrm{Nd}^{3+}$ ion as a new sensitizer into the conventional $\mathrm{Yb}^{3+}$-doped UCNs, which can shift the $980 \mathrm{~nm}$ excitation to a more biocompatible spectral window at $\sim 800 \mathrm{~nm}$. It is well known that water absorption in the wavelength range of $808 \mathrm{~nm}$ is much lower than that approximately $980 \mathrm{~nm}^{41}$, which consequently minimizes the tissue-heating effect during the process of NIR light irradiation. Another 


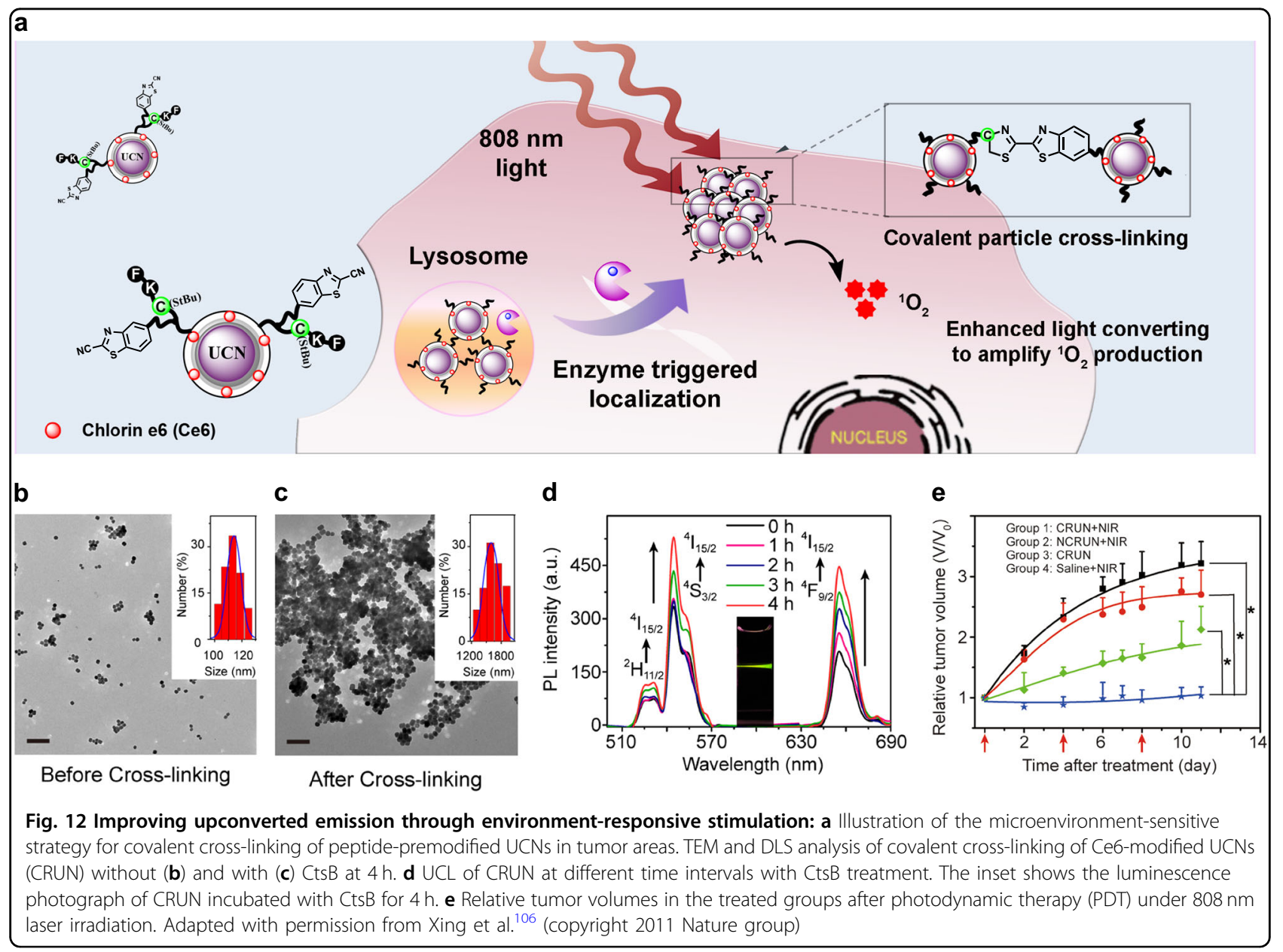

advantage of $\mathrm{Nd}^{3+}$ ion is its large absorption cross-section at $800 \mathrm{~nm}$, which enables enhancement of upconversion luminescence efficiency ${ }^{109}$. So far, a variety of $\mathrm{Nd}^{3+}$-sensitized UCNs have been explored for biomedical applications $^{110-114}$. For example, Yan and coworkers developed $\mathrm{NaGdF}_{4}: \mathrm{Yb}, \quad \mathrm{Er} @ \mathrm{NaGdF}_{4}: \mathrm{Nd}, \mathrm{Yb}$ core-shell nanoparticles for in vivo imaging. Extensive in vitro and in vivo studies demonstrated that the laser-induced tissue overheating effect was greatly minimized under $808 \mathrm{~nm}$ excitation (Fig. 13a) ${ }^{40}$. To effectively eliminate the deleterious cross-relaxation between the activator and sensitizer $\mathrm{Nd}^{3+}$, Zhong et al. have designed a quenching-shield sandwich structure $\left(\mathrm{NaYF}_{4}: \mathrm{Yb}, \mathrm{Er} @ \mathrm{NaYF}_{4}: \mathrm{Yb} @ \mathrm{NaNdF}_{4}\right.$ : $\mathrm{Yb})$ for $800 \mathrm{~nm}$ laser-mediated upconversion luminescence in $\mathrm{Nd}^{3+}$-sensitized nanoparticles (Fig. 13b). In this system, the designed intermediate shell, $\mathrm{NaYF}_{4}: \mathrm{Yb}$, successfully blocks the non-radiative energy transfer and relaxation between $\mathrm{Er}^{3+}$ and $\mathrm{Nd}^{3+}$. By optimizing the thickness of the interlayer, the emission intensity of the system upon $800 \mathrm{~nm}$ excitation reaches a maximum when the interlayer thickness is controlled at approximately $1.45 \mathrm{~nm}$. Furthermore, use of the $800 \mathrm{~nm}$ laser source instead of a $980 \mathrm{~nm}$ laser can greatly increase the penetration depth into biological tissue and minimize unwanted thermal effect ${ }^{109}$.

A second effective strategy to minimize the thermal effect associated with conventional $980 \mathrm{~nm}$ excitation is to employ UCNs with organic fluorophores as sensitizers. As mentioned before, organic dyes have a broader absorption band and larger absorbance cross-section than those of inorganic lanthanide ions, and thus, not only increase the light-harvesting ability of UCNs but also enable extension of NIR laser excitation from the wavelength at $980 \mathrm{~nm}$ to a more biocompatible range (e.g., at $800 \mathrm{~nm}$ ). Upon $800 \mathrm{~nm}$ excitation of such dye-sensitized UCNs, the photothermal effect can be assumed to be largely minimized mainly due to the lower absorbance by water at a spectral window of $808 \mathrm{~nm}$. Therefore, the rational design of dye-sensitized UCNs that can respond to $808 \mathrm{~nm}$ laser illumination could work as an alternative strategy to dramatically decrease the unnecessary thermal effect, and thus provide great feasibility for their improved biomedical applications in living systems. Notably, during the process of laser excitation of dye-sensitized UCNs at 


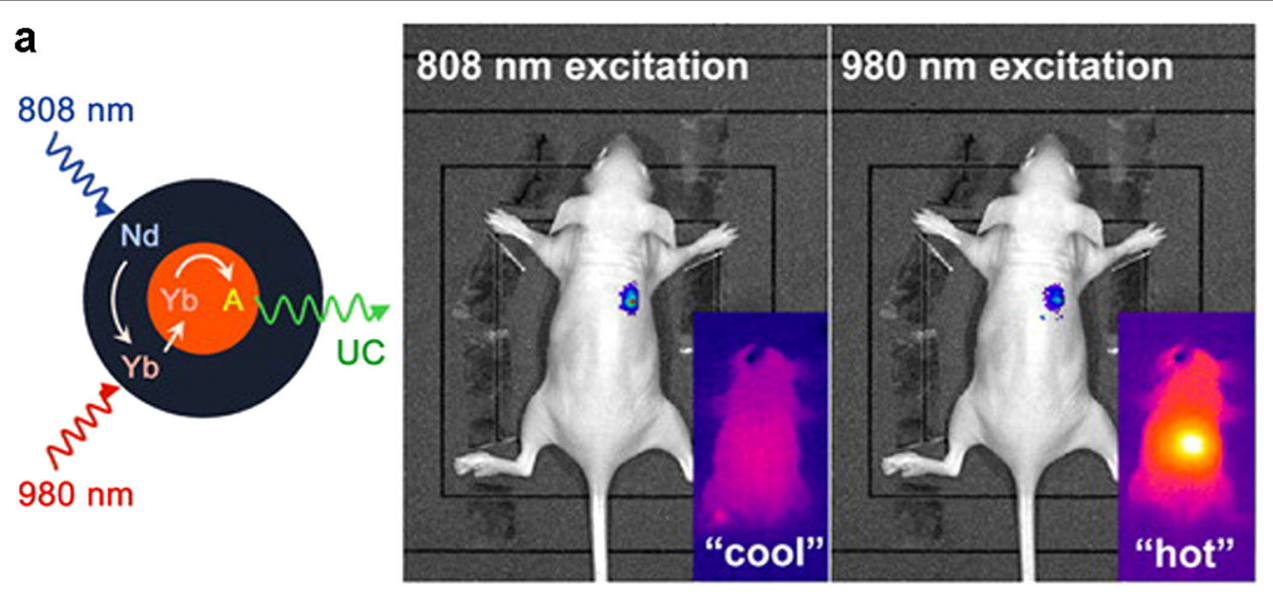

b

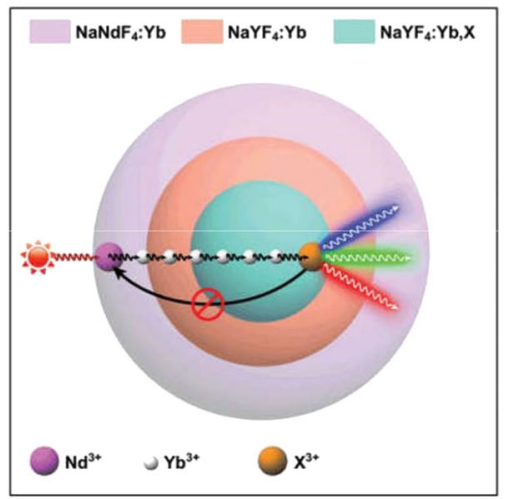

C

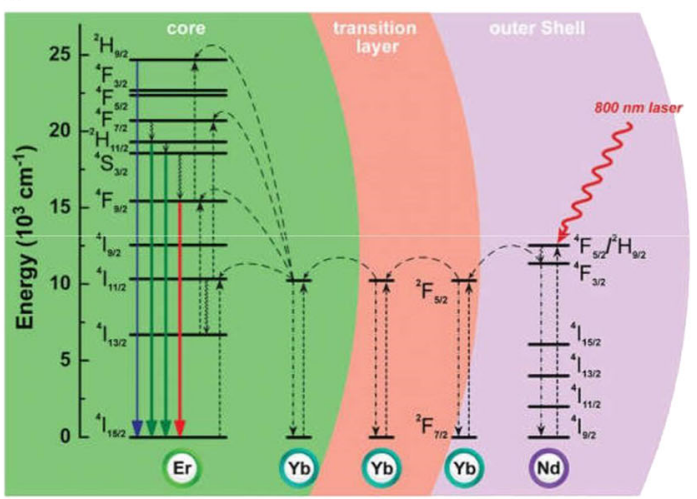

Fig. 13 Minimizing the tissue-heating effect through new sensitizer ion $\mathbf{N d}_{\mathbf{3}}$ : a Scheme of the $\mathrm{Nd}^{3+}-\mathrm{Yb}^{3+}$-activator energy-transfer process by introducing $\mathrm{Nd}^{3+} / \mathrm{Yb}^{3+}$ co-doped shell and in vivo UC imaging of a nude mouse subcutaneously injected with Er@Nd NPs. Laser irradiation with lasers at 980 and $808 \mathrm{~nm}$, both with a power density of $200 \mathrm{~mW} / \mathrm{cm}^{2}$. Adapted with permission from Wang et al. ${ }^{40}$ (copyright 2013 American Chemical Society). b Schematic illustration of the proposed energy-transfer mechanisms in the quenching-shield sandwich-structured UCNs upon $800 \mathrm{~nm}$ excitation. c Proposed energy-transfer mechanisms in the quenching-shield sandwich nanoparticle upon $800 \mathrm{~nm}$ diode-laser excitation. Adapted with permission from Zhong et al. ${ }^{109}$ (copyright 2014 WILEY-VCH Verlag GmbH \& Co. KGaA, Weinheim)

$808 \mathrm{~nm}$, care needs to be taken to assure the proper surface modification on the particle surface, as the stability of organic fluorophore-conjugated nanoparticles would be a key factor in their practical application in living systems ${ }^{115}$.

Apart from optimizing the excitation to a more biocompatible spectral range to alleviate laser-induced heating effects, the excitation power density may also be a potential factor to consider during light irradiation. Typically, upconversion is a type of nonlinear optical process that converts a lower-energy excitation light into a higher energy emission through sequential absorption of multiple excitation photons. In this nonlinear process, the upconversion emission intensity is highly related to the excitation power density ${ }^{116,117}$. Currently, the commercial continuous-wave $(\mathrm{CW})$ laser is the most frequently used excitation source for lanthanide-doped UCNs. In most cases, high $\mathrm{CW}$ excitation power densities are often required to obtain the necessary upconversion emission intensity for potential biomedical applications. However, the high power dosage of laser irradiation could produce substantial heating, which in turn causes undesirable cell/ tissue damage. Thus, it is worthwhile to investigate effective approaches to decrease the laser power density and its accompanying heating effects while guaranteeing the desired emission intensity. To this end, quasicontinuous-wave (quasi-CW, or pulsed) excitation thus offers a good opportunity to achieve the expected upconversion luminescence without concerns about unnecessary heating effects caused by the long duration of NIR laser excitation. Using the equivalent average power density, such quasi-CW excitation has been demonstrated to produce higher quantum yields than does the commonly used CW laser, mainly attributed to the promising capability of quasi-CW excitation with a higher peak power to pump more excitation energy into multiphoton 
emitting channels ${ }^{118,119}$. Due to the much higher pumping efficiency of quasi-CW excitation for multiphoton upconversion emissions, the required average excitation power density is expected to decrease tremendously, thus attenuating local heating from the laser ${ }^{120}$. By adopting this excitation approach, Zhang and coworkers have demonstrated the use of quasi-CW excitation for UCNbased optogenetic manipulation in vitro and in C. elegans. In their work, excitation with a high-power CW NIR laser enabled photoactivation of opsins but induced undesirable heating effects and cell death. However, upon excitation with the quasi-CW laser, the efficient activation of opsins could be still obtained at much lower average powers but with no observable toxicity and heating effects ${ }^{119}$.

\section{Summary and perspectives}

So far, lanthanide-doped UCNs, emerging as a new type of promising luminescent nanomaterial, have been rapidly developed in recent years mainly due to their unique optical features such as large anti-Stokes shifts, narrow emission bands, and high resistance to photobleaching. Particularly, they are capable of converting longwavelength NIR light into short-wavelength emission via sequential absorption of two or more photons. By taking advantage of these distinct optical properties of $\mathrm{Ln}^{3+}$ together with deep tissue penetration and low autofluorescence under NIR laser excitation, a variety of $\mathrm{Ln}^{3+}$ doped UCNs have found applications in diverse biomedical fields including bioimaging, targeted drug/gene delivery, remote activation of biomolecules, and NIR light-controlled therapeutics. Despite the great prospects of UCNs, their low upconversion emission efficiency and laser-induced tissue damage remain as technical challenges, thus remarkably impeding their biomedical applications. In this review, we summarize the recent efforts in boosting the upconversion efficiency of UCNs and suppressing laser-induced overheating effects. These strategies have opened new opportunities for the use of upconverting nanomaterials in a variety of fields. In line with the great efforts in resolving the challenge of upconversion luminescence efficiency, some other aspects and the further development of UCNs towards applications in biomedical and pre-clinical practice still need to be extensively exploited.

First, a fundamental and comprehensive understanding of the optical properties of UCNs will be of great importance for practical application of UCNs. In this regard, development of accurate theoretical modeling that is capable of comprehensively interpreting all processes associated with UCL and predicting the upconversion behavior of a lanthanide-doped $U C N$ is a promising strategy. Such models would provide valuable assistance for the design of upconversion nanosystems and may also help identify novel upconversion patterns. In addition to deriving theoretical models, the development of commercial instruments for the characterization of the photo-properties of UCNs is also crucial for the application of UCNs. Most of the research groups currently use custom-built equipment because lanthanidedoped nanomaterials require specialized excitation sources, which are normally not available in commercial instruments. However, the laser excitation power density and the laser beam profile of custom-built instruments are typically not well characterized, which may further obstruct the potential clinical translation of upconverting nanomaterials.

So far, lanthanide-based UCNs have held great potential in biomedical fields. However, the currently used NIR excitation for UCNs mainly focuses on $800 \mathrm{~nm}$ and $980 \mathrm{~nm}$ irradiation. Considering the complexity of living systems and biological processes being highly dynamic and heterogeneous, it is imperative to broaden the excitation spectrum for spatiotemporal regulation of upconversion luminescence, thereby expanding the scope of application for UCNs. To this end, effective integration of organic dye molecules with UCNs is the preferred choice because organic dyes exhibit much broader absorption bands and larger absorption cross-sections compared with inorganic lanthanide ions. Currently, the photostability of these dye molecules and solubility of dye-sensitized UCNs remain as challenges. Therefore, new chemistry advances including synthesis of organic dyes with excellent photostability and extensive absorption spectra as well as their effective integration within the structures of UCNs are urgently demanded. Additionally, designs of new optical transmission devices or optics fibers will provide good strategies to effectively direct light irradiation into the desired areas located deep inside the body. Such optical devices would be beneficial for precision medicine in real-time tracking of the therapeutic reagents and simultaneous monitoring of the disease surroundings during the treatment.

Benefiting from the rapid progress of nanotechnology, different types of nanomaterials have their respective photophysical properties. Construction of novel heterostructures by integrating UCNs with other materials to make full use of their properties may open up new avenues for their application. For example, construction of NIR-activated upconverted persistent luminescent nanomaterials by combining upconversion and persistent luminescence properties into a single host provides opportunity for long-term in vivo imaging. Moreover, considering the applications of UCNs in biological species, the biocompatibility, stability, and long-term toxicity of UCNs need to be systematically and comprehensively investigated for further pre-clinical and clinical applications. In spite of several surface-modification approaches 
that have successfully improved the biocompatibility and stability of UCNs, the long-term safety in clinical usage remains a challenge. Thus, development of biocompatible and biodegradable upconversion nanomaterials is still highly needed.

\section{Acknowledgements}

This work was partially supported by NTU-AIT-MUV NAM/16001, Tier 1 RG110/ 16 (S), (RG 11/13) and (RG 35/15) awarded in Nanyang Technological University, Singapore and National Natural Science Foundation of China (NSFC) (No. 51628201).

\section{Author details}

${ }^{1}$ Division of Chemistry and Biological Chemistry, School of Physical \& Mathematical Sciences, Nanyang Technological University, Singapore 637371, Singapore. '2 College of Chemistry, Fuzhou University, Fuzhou, Fujian 350116, China. ${ }^{3}$ State Key Laboratory of Rare Earth Resource Utilization, Changchun Institute of Applied Chemistry, Chinese Academy of Sciences, Changchun 130022, China

\section{Conflict of interest}

The authors declare that they have no conflict of interest.

\section{Publisher's note}

Springer Nature remains neutral with regard to jurisdictional claims in published maps and institutional affiliations.

Received: 30 January 2018 Revised: 21 May 2018 Accepted: 27 May 2018. Published online: 1 August 2018

\section{References}

1. Rubin, E. H., Allen, J. D., Nowak, J. A. \& Bates, S. E. Developing precision medicine in a global world. Clin. Cancer Res. 20, 1419-1427 (2014).

2. Friedman, A. A., Letai, A., Fisher, D. E. \& Flaherty, K. T. Precision medicine for cancer with next-generation functional diagnostics. Nat. Rev. Cancer 15, 747-756 (2015).

3. Arnedos, M. et al. Precision medicine for metastatic breast cancer-limitations and solutions. Nat. Rev. Clin. Oncol. 12, 693-704 (2015).

4. Hussain, T. \& Nguyen, Q. T. Molecular imaging for cancer diagnosis and surgery. Adv. Drug Deliv. Rev. 66, 90-100 (2014).

5. Kircher, M. F., Hricak, H. \& Larson, S. M. Molecular imaging for personalized cancer care. Mol. Oncol. 6, 182-195 (2012).

6. Kuimova, M. K. et al. Imaging intracellular viscosity of a single cell during photoinduced cell death. Nat. Chem. 1, 69-73 (2009).

7. Weissleder, R., Schwaiger, M. C., Gambhir, S. S. \& Hricak, H. Imaging approaches to optimize molecular therapies. Sci. Transl. Med. 8, 355ps316 (2016).

8. Yang, Y. et al. Core-shell and co-doped nanoscale metal-organic particles (NMOPs) obtained via post-synthesis cation exchange for multimodal imaging and synergistic thermo-radiotherapy. NPG Asia Mater. 9, e344-e344 (2017).

9. Willmann, J. K., van Bruggen, N., Dinkelborg, L. M. \& Gambhir, S. S. Molecular imaging in drug development. Nat. Rev. Drug. Discov. 7, 591-607 (2008).

10. Kobayashi, H., Ogawa, M., Alford, R., Choyke, P. L. \& Urano, Y. New strategies for fluorescent probe design in medical diagnostic imaging. Chem. Rev. 110, 2620-2640 (2010).

11. Rao, J., Dragulescu-Andrasi, A. \& Yao, H. Fluorescence imaging in vivo: recent advances. Curr. Opin. Biotechnol. 18, 17-25 (2007)

12. Medintz, I. L., Uyeda, H. T., Goldman, E. R. \& Mattoussi, H. Quantum dot bioconjugates for imaging, labelling and sensing. Nat. Mater. 4, 435-446 (2005).

13. Wegner, K. D. \& Hildebrandt, N. Quantum dots: bright and versatile in vitro and in vivo fluorescence imaging biosensors. Chem. Soc. Rev. 44, 4792-4834 (2015)
14. Gao, M., Yu, F., LV, C., Choo, J. \& Chen, L. Fluorescent chemical probes for accurate tumor diagnosis and targeting therapy. Chem. Soc. Rev. 46 2237-2271 (2017).

15. Chen, Z. J., Ren, W., Wright, Q. E. \& Ai, H. W. Genetically encoded fluorescent probe for the selective detection of peroxynitrite. J. Am. Chem. Soc. 135, 14940-14943 (2013)

16. Heil, K., Pearson, D. \& Carell, T. Chemical investigation of light induced DNA bipyrimidine damage and repair. Chem. Soc. Rev. 40, 4271-4278 (2011).

17. Yang, Y., Mu, J. \& Xing, B. Photoactivated drug delivery and bioimaging. Wiley Interdiscip. Rev. Nanomed. Nanobiotechnol. 9, e1408 (2017).

18. Yang, Y., Aw, J. \& Xing, B. Nanostructures for NIR light-controlled therapies. Nanoscale 9, 3698-3718 (2017).

19. Hilderbrand, S. A. \& Weissleder, R. Near-infrared fluorescence: application to in vivo molecular imaging. Curr. Opin. Chem. Biol. 14, 71-79 (2010).

20. Idris, N. M., Jayakumar, M. K., Bansal, A. \& Zhang, Y. Upconversion nanoparticles as versatile light nanotransducers for photoactivation applications. Chem. Soc. Rev. 44, 1449-1478 (2015).

21. Yang, D. et al. Current advances in lanthanide ion ( $\operatorname{Ln}(3+))$-based upconversion nanomaterials for drug delivery. Chem. Soc. Rev. 44, 1416-1448 (2015).

22. Zheng, W. et al. Lanthanide-doped upconversion nano-bioprobes: electronic structures, optical properties, and biodetection. Chem. Soc. Rev. 44, 1379-1415 (2015).

23. Chen, C., Li, C. \& Shi, Z. Current advances in lanthanide-doped upconversion nanostructures for detection and bioapplication. Adv. Sci. 3, 1600029 (2016).

24. Liu, G. Advances in the theoretical understanding of photon upconversion in rare-earth activated nanophosphors. Chem. Soc. Rev. 44, 1635-1652 (2015).

25. Han, S., Deng, R., Xie, X. \& Liu, X. Enhancing luminescence in lanthanidedoped upconversion nanoparticles. Angew. Chem. Int. Ed. 53, 11702-11715 (2014).

26. Biju, $\mathrm{V}$. Chemical modifications and bioconjugate reactions of nanomaterials for sensing, imaging, drug delivery and therapy. Chem. Soc. Rev. 43, 744-764 (2014)

27. Chen, G., Qiu, H., Prasad, P. N. \& Chen, X. Upconversion nanoparticles: design, nanochemistry, and applications in theranostics. Chem. Rev. 114, 5161-5214 (2014).

28. Feng, W., Zhu, X. \& Li, F. Recent advances in the optimization and functionalization of upconversion nanomaterials for in vivo bioapplications. NPG Asia Mater. 5, e75 (2013)

29. Xing, $\mathrm{H}$. et al. Multifunctional nanoprobes for upconversion fluorescence, MR and CT trimodal imaging. Biomaterials 33, 1079-1089 (2012).

30. Shi, J. et al. A new near-infrared persistent luminescence nanoparticle as a multifunctional nanoplatform for multimodal imaging and cancer therapy. Biomaterials 152, 15-23 (2018).

31. Sun, $Y$. et al. Fluorine-18 labeled rare-earth nanoparticles for positron emission tomography (PET) imaging of sentinel lymph node. Biomaterials 32, 2999-3007 (2011).

32. Yang, Y. et al. Hydrothermal synthesis of $\mathrm{NaLuF}_{4} \cdot{ }^{153} \mathrm{Sm}, \mathrm{Yb}, \mathrm{Tm}$ nanoparticles and their application in dual-modality upconversion luminescence and SPECT bioimaging. Biomaterials 34, 774-783 (2013).

33. Zhou, J., Liu, Z. \& Li, F. Upconversion nanophosphors for small-animal imaging. Chem. Soc. Rev. 41, 1323-1349 (2012).

34. Min, Y., Li, J., Liu, F., Yeow, E. K. \& Xing, B. Near-infrared light-mediated photoactivation of a platinum antitumor prodrug and simultaneous cellular apoptosis imaging by upconversion-luminescent nanoparticles. Angew. Chem. Int. Ed. 53, 1012-1016 (2014).

35. Yang, Y. et al. In vitro and in vivo uncaging and bioluminescence imaging by using photocaged upconversion nanoparticles. Angew. Chem. Int. Ed. $\mathbf{5 1}$ 3125-3129 (2012)

36. $\mathrm{Ai}, \mathrm{X}$. et al. Remote regulation of membrane channel activity by site-specific localization of lanthanide-doped upconversion nanocrystals. Angew. Chem. Int. Ed. 56, 3031-3035 (2017).

37. Peng, J. et al. Real-time in vivo hepatotoxicity monitoring through chromophore-conjugated photon-upconverting nanoprobes. Angew. Chem. Int. Ed. 56, 4165-4169 (2017).

38. Su, Q., Feng, W., Yang, D. \& Li, F. Resonance energy transfer in upconversion nanoplatforms for selective biodetection. Acc. Chem. Res. 50, 32-40 (2017).

39. Chen, G., Agren, H., Ohulchanskyy, T. Y. \& Prasad, P. N. Light upconverting core-shell nanostructures: nanophotonic control for emerging applications. 
Chem. Soc. Rev. 44, 1680-1713 (2015).

40. Wang, Y. F. et al. $\mathrm{Nd}^{3+}$-Sensitized upconversion nanophosphors: efficient in vivo bioimaging probes with minimized heating effect. ACS Nano 7, 7200-7206 (2013).

41. Shen, J. et al. Engineering the upconversion nanoparticle excitation wavelength: cascade sensitization of tri-doped upconversion colloidal nanoparticles at $800 \mathrm{~nm}$. Adv. Opt. Mater. 1, 644-650 (2013).

42. Haase, M. \& Schafer, H. Upconverting nanoparticles. Angew. Chem. Int. Ed. 50, 5808-5829 (2011).

43. Auzel, F. Upconversion and anti-stokes processes with $\mathrm{f}$ and $\mathrm{d}$ ions in solids. Chem. Rev. 104, 139-173 (2004).

44. Dong, H., Sun, L. D. \& Yan, C. H. Basic understanding of the lanthanide related upconversion emissions. Nanoscale 5, 5703-5714 (2013)

45. Dong, A. et al. A generalized ligand-exchange strategy enabling sequential surface functionalization of colloidal nanocrystals. J. Am. Chem. Soc. 133 998-1006 (2011)

46. Duan, C., Liang, L., Li, L., Zhang, R. \& Xu, Z. P. Recent progress in upconversion luminescence nanomaterials for biomedical applications. J. Mater. Chem. B 6 192-209 (2018).

47. Huang, L. et al. Expanding anti-stokes shifting in triplet-triplet annihilation upconversion for in vivo anticancer prodrug activation. Angew. Chem. Int. Ed. Engl. 56, 14400-14404 (2017).

48. Huang, L. et al. Enhancing photodynamic therapy through resonance energy transfer constructed near-infrared photosensitized nanoparticles. Adv. Mater. 291604789 (2017)

49. Bai, Y. et al. The effect of $\mathrm{Li}$ on the spectrum of $\mathrm{Er}^{3+}$ in $\mathrm{Li}$-and Er-codoped ZnO nanocrystals. J. Phys. Chem. C. 112, 12259-12263 (2008).

50. Luo, X. \& Cao, W. Upconversion luminescence properties of $\mathrm{Li}^{+}$-doped $\mathrm{ZnWO}_{4}$ : Yb, Er. J. Mater. Res. 23, 2078-2083 (2008).

51. Cao, B., Feng, Z., He, Y., Li, H. \& Dong, B. Opposite effect of Li+ codoping on the upconversion emissions of $\mathrm{Er}^{3+}$-doped $\mathrm{TiO}_{2}$ powders. J. Sol-Gel Sci. Technol. 54, 101-104 (2010).

52. Liu, L. et al. Efficient two-color luminescence of $\mathrm{Er}^{3+} \mathrm{Mb}^{3+} / \mathrm{Li}^{+} \mathrm{ZrO}_{2}$ nanocrystals. Opt. Mater. 33, (1234-1238 (2011).

53. Yin, W. et al. Enhanced red emission from $\mathrm{GdF}_{3}: \mathrm{Yb}^{3+}, \mathrm{Er}^{3+}$ upconversion nanocrystals by $\mathrm{Li}^{+}$doping and their application for bioimaging. Chem. Eur. J. 18, 9239-9245 (2012).

54. $\mathrm{Hu}, \mathrm{M}$. et al. Synergistically enhanced upconversion luminescence in $\mathrm{Li}^{+}-$ doped core-shell-structured ultrasmall nanoprobes for dual-mode deep tissue fluorescence/CT imaging. J. Mater. Chem. B 5, 2662-2670 (2017).

55. Liang, $\mathrm{H}$. et al. Enhancement of upconversion luminescence of $\mathrm{Y}_{2} \mathrm{O}_{3}: \mathrm{Er}^{3+}$ nanocrystals by codoping $\mathrm{Li}^{+}-\mathrm{Zn}^{2+}$. J. Alloy. Compd. 509, 409-413 (2011).

56. Jiang, L., Xiao, S., Yang, X., Ding, J. \& Dong, K. Enhancement of up-conversion luminescence in $\mathrm{Zn}_{2} \mathrm{SiO}_{4}: \mathrm{Yb}^{3+}, \mathrm{Er}^{3+}$ by co-doping with $\mathrm{Li}^{+}$or $\mathrm{Bi}^{3+}$. Appl. Phys. B 107, 477-481 (2012).

57. Niu, N. et al. Rapid microwave reflux process for the synthesis of pure hexagonal $\mathrm{NaYF}_{4}$ : $\mathrm{Yb}^{3+}, \mathrm{Ln}^{3+}, \mathrm{Bi}^{3+}\left(\mathrm{Ln}^{3+}=\mathrm{Er}^{3+}, \mathrm{Tm}^{3+}, \mathrm{Ho}^{3+}\right)$ and its enhanced UC luminescence. J. Mater. Chem. 22, 21613-21623 (2012).

58. Ramasamy, P., Chandra, P., Rhee, S. W. \& Kim, J. Enhanced upconversion luminescence in $\mathrm{NaGdF}_{4}$ : $\mathrm{Yb}$, Er nanocrystals by $\mathrm{Fe}^{3+}$ doping and their application in bioimaging. Nanoscale 5, 8711-8717 (2013).

59. Auzel, F. Upconversion and anti-stokes processes with $\mathrm{f}$ and $\mathrm{d}$ ions in solids. Chem. Rev. 104, 139-174 (2004)

60. Zhao, J. et al. Single-nanocrystal sensitivity achieved by enhanced upconversion luminescence. Nat. Nanotechnol. 8, 729-734 (2013).

61. Wang, J. et al. Enhancing multiphoton upconversion through energy clustering at sublattice level. Nat. Mater. 13, nmat3804 (2013).

62. Cao, B., He, Y., Feng, Z., Li, Y. \& Dong, B. Optical temperature sensing behavior of enhanced green upconversion emissions from $\mathrm{Er}-\mathrm{Mo}: \mathrm{Yb}_{2} \mathrm{Ti}_{2} \mathrm{O}_{7}$ nanophosphor. Sens. Actuator B-Chem. 159, 8-11 (2011).

63. Dong, B. et al. Temperature sensing and in vivo imaging by molybdenum sensitized visible upconversion luminescence of rare-earth oxides. Adv. Mater. 24, 1987-1993 (2012).

64. Li, Z., Dong, B., He, Y., Cao, B. \& Feng, Z. Selective enhancement of green upconversion emissions of $\mathrm{Er}^{3+}{ }^{3+} \mathrm{Yb}^{3+} \mathrm{Al}_{5} \mathrm{O}_{12}$ nanocrystals by high excited state energy transfer with $\mathrm{Yb}^{3+}-\mathrm{Mn}^{2+}$ dimer sensitizing. J. Lumin. 132, 1646-1648 (2012).

65. Li, X. et al. Energy migration upconversion in manganese (II)- doped nanoparticles. Angew. Chem. Int. Ed. 127, 13510-13515 (2015).
66. Lingeshwar Reddy, K. et al. Enhancement of luminescence intensity in red emitting $\mathrm{NaYF}_{4}$ : $\mathrm{Yb} / \mathrm{Ho} / \mathrm{Mn}$ upconversion nanophosphors by variation of reaction parameters. J. Phys. Chem. C 121, 11783-11793 (2017).

67. Zeng, J. H., Xie, T., Li, Z. H. \& Li, Y. Monodispersed nanocrystalline fluoroperovskite up-conversion phosphors. Cryst. Growth Des. 7, 2774-2777 (2007).

68. Xie, M.Y. et al. Synthesis of $\mathrm{Yb}^{3+} / \mathrm{Er}^{3+}$ co-doped $\mathrm{MnF}_{2}$ nanocrystals with bright red up-converted fluorescence. Scr. Mater. 60, 190-193 (2009).

69. Wang, J., Wang, F., Wang, C., Liu, Z. \& Liu, X. Single-band upconversion emission in lanthanide-doped $\mathrm{KMnF}_{3}$ nanocrystals. Angew. Chem. Int. Ed. $\mathbf{5 0}$ 10369-10372 (2011).

70. Zhang, Y., Lin, J. D., Vijayaragavan, V., Bhakoo, K. K. \& Tan, T. T. Y. Tuning sub-10 $\mathrm{nm}$ single-phase $\mathrm{NaMnF}_{3}$ nanocrystals as ultrasensitive hosts for pure intense fluorescence and excellent $\mathrm{T} 1$ magnetic resonance imaging. Chem. Commun. 48, 10322-10324 (2012).

71. Tian, G. et al. $\mathrm{Mn}^{2+}$ dopant-controlled synthesis of $\mathrm{NaYF}_{4}$ : Yb/Er upconversion nanoparticles for in vivo imaging and drug delivery. Adv. Mater. 24, 1226-1231 (2012).

72. Wang, F., Fan, X., Wang, M. \& Zhang, Y. Multicolour PEI/NaGdF $4: \mathrm{Ce}^{3+}, \mathrm{Ln}^{3+}$ nanocrystals by single-wavelength excitation. Nanotechnology 18, 025701 (2006).

73. Zhou, J. et al. Fluorine-18-labeled $\mathrm{Gd}^{3+} / \mathrm{Nb}^{3+} / \mathrm{Er}^{3+}$ co-doped $\mathrm{NaYF}_{4}$ nanophosphors for multimodality PET/MR/UCL imaging. Biomaterials 32 1148-1156 (2011).

74. Chen, Q. et al. Confining excitation energy in $\mathrm{Er}^{3+}$-sensitized upconversion nanocrystals through $\mathrm{Tm}^{3+}$-Mediated transient energy trapping. Angew. Chem. Int. Ed. 56, 7605-7609 (2017).

75. Yi, G. \& Chow, G. Water-soluble NaYF : Yb,Er(Tm)/NaYF//polymer core/shell/ shell nanoparticles with significant enhancement of upconversion fluorescence. Chem. Mater. 19, 341-343 (2007).

76. Mai, H., Zhang, Y., Sun, L. \& Yan, C. Highly efficient multicolor up-conversion emissions and their mechanisms of monodisperse $\mathrm{NaYF}_{4}$ : $\mathrm{Yb}, \mathrm{Er}$ core and core/shell-structured nanocrystals. J. Phys. Chem. C. 111, 13721-13729 (2007).

77. Schäfer, H., Ptacek, P., Zerzouf, O. \& Haase, M. Synthesis and optical properties of $\mathrm{KYF}_{4} \mathrm{Yb}$, Er nanocrystals, and their surface modification with undoped $\mathrm{KYF}_{4}$. Adv. Funct. Mater. 18, 2913-2918 (2008).

78. Ansari, A. A., Yadav, R. \& Rai, S. Enhanced luminescence efficiency of aqueous dispersible $\mathrm{NaYF}_{4}: \mathrm{Yb} / \mathrm{Er}$ nanoparticles and the effect of surface coating. RSC Adv. 6, 22074-22082 (2016).

79. Dong, C., Korinek, A., Blasiak, B., Tomanek, B. \& van Veggel, F. C. Cation

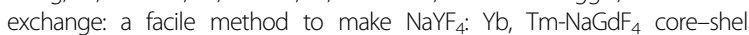
nanoparticles with a thin, tunable, and uniform shell. Chem. Mater. 24 1297-1305 (2012).

80. Lin, X. et al. Core-shell-shell upconversion nanoparticles with enhanced emission for wireless optogenetic inhibition. Nano. Lett. 18, 948-956 (2018).

81. Vetrone, F., Naccache, R., Mahalingam, V., Morgan, C. G. \& Capobianco, J. A. The active-core/active-shell approach: A strategy to enhance the upconversion luminescence in lanthanide-doped nanoparticles. Adv. Funct. Mater. 19, 2924-2929 (2009).

82. Qian, H.S. \& Zhang, Y. Synthesis of hexagonal-phase core-shell NaYF nanocrystals with tunable upconversion fluorescence. Langmuir $\mathbf{2 4}$ 12123-12125 (2008).

83. Ghosh, P. et al. Enhancement of upconversion emission of LaPO4: Er@ Yb core-shell nanoparticles/nanorods. J. Phys. Chem. C. 112, 9650-9658 (2008).

84. Liu, Y. et al. A strategy to achieve efficient dual-mode luminescence of $\mathrm{Eu}^{3+}$ in lanthanides doped multifunctional $\mathrm{NaGdF}_{4}$ nanocrystals. Adv. Mater. 22, 3266-3271 (2010).

85. Liu, X. et al. Breakthrough in concentration quenching threshold of upconversion luminescence via spatial separation of the emitter doping area for bio-applications. Chem. Commun. 47, 11957-11959 (2011).

86. Yang, D. et al. Colloidal synthesis and remarkable enhancement of the upconversion luminescence of $\mathrm{BaGdF}_{5}: \mathrm{Yb}^{3+} / \mathrm{Er}^{3+}$ nanoparticles by activeshell modification. J. Mater. Chem. 21, 5923-5927 (2011).

87. Chen, D. et al. Lanthanide dopant-induced formation of uniform sub-10 nm active-core/active-shell nanocrystals with near-infrared to near-infrared dualmodal luminescence. J. Mater. Chem. 22, 2632-2640 (2012).

88. Zhang, Y. et al. Synthesis of ultra-small BaLuF 5 : Yb ${ }^{3+}, \mathrm{Er}^{3+} @$ BaLuF $_{5}$ : Yb ${ }^{3+}$ active-core-active-shell nanoparticles with enhanced up-conversion and 
down-conversion luminescence by a layer-by-layer strategy. J. Mater. Chem. C. 3, 2045-2053 (2015).

89. Wang, F. et al. Tuning upconversion through energy migration in core-shell nanoparticles. Nat. Mater. 10, 968-973 (2011).

90. Chen, X. et al. Confining energy migration in upconversion nanoparticles towards deep ultraviolet lasing. Nat. Commun. 7, 10304 (2016).

91. Jin, L. M., Chen, X., Siu, C. K., Wang, F. \& Yu, S. F. Enhancing multiphoton upconversion from $\mathrm{NaYF}_{4}: \mathrm{Yb} / \mathrm{Tm} @ \mathrm{NaYF}_{4}$ core-shell nanoparticles via the use of laser cavity. ACS Nano 11, 843-849 (2017).

92. Barnes, W. L., Dereux, A. \& Ebbesen, T. W. Surface plasmon subwavelength optics. Nature 424, 824-830 (2003).

93. Zhang, $\mathrm{H}$. et al. Plasmonic modulation of the upconversion fluorescence in $\mathrm{NaYF}_{4}: \mathrm{Yb} / \mathrm{Tm}$ hexaplate nanocrystals using gold nanoparticles or nanoshells. Angew. Chem. Int. Ed. 49, 2865-2868 (2010).

94. Feng, A. L. et al. Distance-dependent plasmon-enhanced fluorescence of upconversion nanoparticles using polyelectrolyte multilayers as tunable spacers. Sci. Rep. 5, 7779 (2015).

95. Saboktakin, M. et al. Metal-enhanced upconversion luminescence tunable through metal nanoparticle nanophosphor separation. ACS Nano 6 8758-8766 (2012).

96. Zhang, W., Ding, F. \& Chou, S. Y. Large enhancement of upconversion

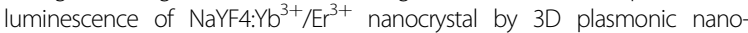
antennas. Adv. Mater. 24, OP236-OP241 (2012).

97. Yin, Z. et al. Plasmon-enhanced upconversion luminescence on vertically aligned gold nanorod monolayer supercrystals. ACS Appl. Mater. Interfaces 8 , 11667-11674 (2016)

98. Niu, W. et al. 3-Dimensional photonic crystal surface enhanced upconversion emission for improved near-infrared photoresponse. Nanoscale 6, 817-824 (2014).

99. Wang, X. et al. Dye-sensitized lanthanide-doped upconversion nanoparticles. Chem. Soc. Rev. 46, 4150-4167 (2017).

100. Chen, G. et al. Energy-cascaded upconversion in an organic dye-sensitized core/shell fluoride nanocrystal. Nano. Lett. 15, 7400-7407 (2015).

101. Zou, W., Visser, C., Maduro, J. A., Pshenichnikov, M. S. \& Hummelen, J. C. Broadband dye-sensitized upconversion of near-infrared light. Nat. Photonics 6. 560-564 (2012).

102. $\mathrm{Wu}, \mathrm{X}$. et al. Tailoring dye-sensitized upconversion nanoparticle excitation bands towards excitation wavelength selective imaging. Nanoscale 7 18424-18428 (2015).

103. Lee, J. et al. Ultra-wideband multi-dye-sensitized upconverting nanoparticles for information security application. Adv. Mater. 29, 1603169 (2017)

104. Wu, X. et al. Dye-sensitized core/active shell upconversion nanoparticles for optogenetics and bioimaging applications. ACS Nano 10, 1060-1066 (2016)

105. Zou, X. et al. A water-dispersible dye-sensitized upconversion nanocomposite modified with phosphatidylcholine for lymphatic imaging. Chem. Commun. 52, 13389-13392 (2016).
106. $\mathrm{Ai}, \mathrm{X}$. et al. In vivo covalent cross-linking of photon-converted rare-earth nanostructures for tumour localization and theranostics. Nat. Comm. 7, 10432 (2016).

107. Liras, M. et al. Thin amphiphilic polymer-capped upconversion nanoparticles: enhanced emission and thermoresponsive properties. Chem. Mater. 26, 4014-4022 (2014)

108. Wang, D. et al. $808 \mathrm{~nm}$ driven $\mathrm{Nd}^{3+}$-sensitized upconversion nanostructures for photodynamic therapy and simultaneous fluorescence imaging. Nanoscale 7, 190-197 (2015).

109. Zhong, Y. et al. Elimination of photon quenching by a transition layer to fabricate a quenching-shield sandwich structure for $800 \mathrm{~nm}$ excited upconversion luminescence of $\mathrm{Nd}^{3+}$-sensitized nanoparticles. $\mathrm{Adv}$. Mater. 26, 2831-2837 (2014)

110. Wang, R., Li, X., Zhou, L. \& Zhang, F. Epitaxial seeded growth of rare-earth nanocrystals with efficient $800 \mathrm{~nm}$ near-infrared to $1525 \mathrm{~nm}$ shortwavelength infrared downconversion photoluminescence for in vivo bioimaging. Angew. Chem. Int. Ed. 53, 12086-12090 (2014).

111. Xie, X. et al. Emerging approximately $800 \mathrm{~nm}$ excited lanthanide-doped upconversion nanoparticles. Small. 13, 1602843 (2017).

112. Zou, X. et al. An $\mathrm{Nd}^{3+}$-sensitized upconversion nanophosphor modified with a cyanine dye for the ratiometric upconversion luminescence bioimaging of hypochlorite. Nanoscale 7, 4105-4113 (2015).

113. Chan, M. H. et al. Minimizing the heat effect of photodynamic therapy based on inorganic nanocomposites mediated by $808 \mathrm{~nm}$ near-infrared light. Small 131700038 (2017).

114. Hou, Z. et al. $808 \mathrm{~nm}$ Light-triggered and hyaluronic acid-targeted dualphotosensitizers nanoplatform by fully utilizing $\mathrm{Nd}(3+)$-sensitized upconversion emission with enhanced anti-tumor efficacy. Biomaterials 101, 32-46 (2016).

115. Wilhelm, S. Perspectives for upconverting nanoparticles. ACS Nano 11, 10644-10653 (2017).

116. Xu, C. T. et al. Upconverting nanoparticles for pre-clinical diffuse optical imaging, microscopy and sensing: current trends and future challenges. Laser Photonics Rev. 7, 663-697 (2013).

117. Liu, H. et al. Photon upconversion kinetic nanosystems and their optical response. Laser Photonics Rev., 12, 1700144 (2017).

118. Liu, H. et al. Deep tissue optical imaging of upconverting nanoparticles enabled by exploiting higher intrinsic quantum yield through use of millisecond single pulse excitation with high peak power. Nanoscale $\mathbf{5}$, 10034-10040 (2013).

119. Bansal, A., Liu, H., Jayakumar, M. K., Andersson-Engels, S. \& Zhang, Y. Quasi-continuous wave near-infrared excitation of upconversion nanoparticles for optogenetic manipulation of C. elegans. Small 12, 1732-1743 (2016).

120. Zhan, Q., He, S., Qian, J., Cheng, H. \& Cai, F. Optimization of optical excitation of upconversion nanoparticles for rapid microscopy and deeper tissue imaging with higher quantum yield. Theranostics 3, 306-316 (2013). 